\title{
ABSOLUTE METHODS IN REFLECTOMETRY
}

\author{
By H. J. McNicholas
}

ABSTRACT

The theory and use of the integrating sphere in three methods of reflectometry, as proposed by Sharp and Little, Karrer, and Taylor, respectively, are discussed in connection with a new absolute method in reflectometry involving no direct use of an integrating device.

The new method is based upon a general law of reciprocity, first stated by Helmholtz, by means of which certain reciprocal relations between the reflective properties for unidirectional and diffused illumination are derived and applied in the method. Under completely diffused illumination the brightness of a sample, in general, increases with increasing angle of observation from the normal to its surface. The illumination on the sample being known, these brightness data are shown to yield (with the aid of the reciprocity law) the reflectance of the sample (ratio of total reflected to total incident light) for any manner of illumination ranging from unidirectional to completely diffused.

Equipment is described for the measurement of reflective properties of materials under either completely diffused or unidirectional illumination for various directions of observation. The samples used include various kinds of materials chosen to cover a wide range of reflectance and to represent various degrees of departure from the perfect diffusor. Comparative measurements by all the methods studied are made on these samples.

\section{CONTENTS}

1. Introduction

II. Definitions and nomenclature-

III. Reciprocal relations ___

IV. New method in reflectometry

V. Theory of the sphere reflectometer 41

1. Methods of Sharp and Little and of Karrer........ 41

2. The Taylor reflectometer 42

VI. Measurement of apparent reflectance

1. Description of samples_............. 44

2. Apparatus and method for diffused illumination

3. Brightness distribution over hemispherical source_._._._. 49

4. Method for unidirectional illumination

5. Calculation of reflectance

VII. Discussion of data

VIII. Use of sphere reflectometer

1. Description of diffused-illumination reflectometer and measurements

2. Evaluation of illumination on sample

3. Errors in measurement of reflected light

4. Design of sphere

5. Measurements with the Taylor reflectometer $\ldots$ 


\section{INTRODUCTION}

In various phases of photometry and colorimetry it is often desirable to obtain an absolute evaluation of some of the reflective or transmissive properties of various diffusing media. The illuminating engineer may require a knowledge of the fraction of incident lightreflected by materials forming the walls and ceiling of a room as an aid in the proper design of lighting installations. The physical stimulus of color is very often the light diffusely reflected (or transmitted) by some material, such as paper, painted surfaces, dyed fabrics, etc. In colorimetric practice it has usually been most convenient to express the reflective (or transmissive) properties of these materials in terms of some material which could be regarded as a standard, but suitable and accurate methods ${ }^{1}$ of proven reliability are needed for the evaluation of the standard (if not the sample itself) in absolute measure - particularly in regard to variations with wave length.

The reflective and transmissive properties of a diffusing medium depend on the nature and structure of the medium, the topography of its bounding surfaces, the angular distribution of the incident luminous flux, and its spectral composition. The absolute evaluation of the reflection or transmission coefficients involves an integration of the incident and of the reflected or transmitted light with respect to all directions from the surface. The photometric integrating sphere (Ulbricht sphere), long used in the photometry of light sources, has been applied in various ways for this purpose. The theory of its use is based, however, upon the simplifying assumption that the material forming the interior surface of the sphere wall reflects incident light in accordance with the cosine law ${ }^{2}$ of emission. It is well known that the reflection of light by all diffusely reflecting materials departs considerably from this law, and that the degree of departure depends to a large extent on the angular and spectral distribution of the incident light. The extent to which the diffuse reflective properties of the sphere wall and sample affect the performance of the sphere is a matter over which there is at present considerable uncertainty.

In this investigation some of the reflective properties of a selected group of samples are exhibited and the data applied to a study of methods used in reflectometry. A thorough analysis of the methods proposed by Sharp and Little ${ }^{3}$ and by Karrer ${ }^{4}$ is made. The relation of the Taylor ${ }^{5}$ method to these is shown, along with comparative

\footnotetext{
1 In the special case of the unidirectional transmission of light by optically homogeneous (nondiffusing) media, accurate and reliable absolute methods are available. See Report of the Optical Society of America, Progress Committee on Spectrophotometry, J. Opt. Soc. Am. and Rev. Sci. Inst., 10, p. 169; 1925.

${ }^{2}$ For a medium obeying the cosine law of emission, the flux in a given direction, from an element of area of the surface, is proportional to the cosine of the angle between the given direction and the normal to the emitting element.

${ }^{3}$ Trans. III. Eng. Soc. $15^{2}$, p. 802; 1920.

${ }^{1}$ B. S. Sci. Paper No. 415; August, 1921. Also J. Opt. Soc. Am. and Rev. Sci. Inst. 5, pp. 96-120; 1921.

${ }^{8}$ Trans. Ill. Eng. Soc., 152. p. 811; 1920. B. S. Sci. Paper No. 405; November, 1920.
} 
measurements by the Taylor reflectometer. A general method of reflectometry is also described, involving no direct use of an integrating device.

\section{DEFINITIONS AND NOMENCLATURE}

Consider the sample in the form of a flat slab illuminated by an extended source subtending, in general, a solid angle $2 \pi$ at the center of the sample. Let $\theta$ and $\phi$ (fig. 1) be the angle with the normal and azimuth angle, respectively, of an incident pencil of light, included in the elementary solid angle $d \omega$; and let $\theta^{\prime}, \phi^{\prime}$, and $d \omega^{\prime}$, be of corresponding import for a reflected pencil. The brightness ${ }^{6}$ distribution over the source, as viewed from the position of the sample, is represented by the function $B(\theta, \phi)$. Similarly, the brightnesses of the

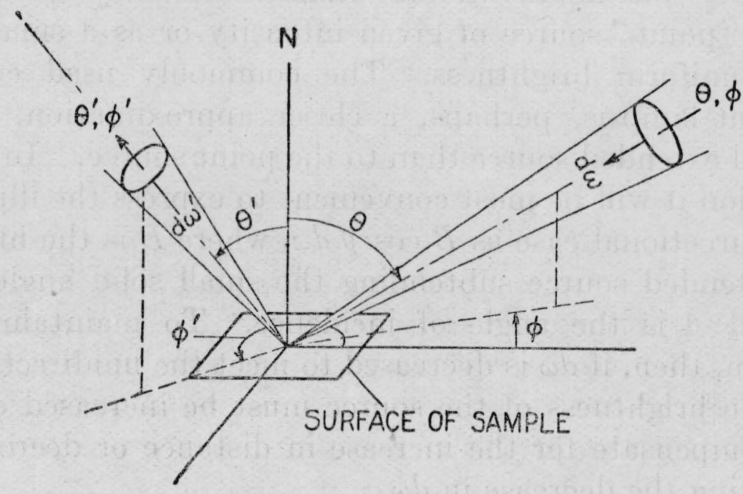

Fig. 1.-Diagram of incident and reflected rays

sample for a given manner of illumination and for various directions of observation $\left(\theta^{\prime}, \phi^{\prime}\right)$ are represented by the function $B^{\prime}\left(\theta^{\prime}, \phi^{\prime}\right)$.

The reflectance ${ }^{7}$ of the sample is defined as the ratio of total reflected to total incident luminous flux (per unit area of surface) and designated by the symbol $R$. Then

$$
R \equiv \frac{\text { Total reflected flux }}{\text { Total incident flux }}=\frac{\mathcal{S} B^{\prime}\left(\theta^{\prime}, \phi^{\prime}\right) \cos \theta^{\prime} d \omega^{\prime}}{\int B(\theta, \phi) \cos \theta d \omega}
$$

where the integrations are taken, in both numerator and denominator, over the solid angle $2 \pi$.

As the reflectance of a given sample is a function of the spectral composition and the angular distribution of the incident flux, it is necessary to define more specifically certain groups of reflectances

\footnotetext{
${ }^{6}$ The brightness of the source, viewed from a given direction is proportional to the luminous flux in that direction per unit of projected area per unit solid angle. See illuminating engineering nomenclature and photometric standards; Trans. Ill. Eng. Soc., 20, p. 629; 1925. In this work we are ultimately concerned with the ratio of brightnesses, hence no particular unit need be specified.

7 This term has been used in the report of the Opt. Soc. Am. Progress Committee for 1922-23, on Spectrophotometry, J. Opt. Soc. Am. and Rev. Sci. Inst., 10, p. 178; 1925.
} $2284^{\circ}-28-3$ 
corresponding to different types of illumination. For the samples used in this investigation the variation of reflectance with spectral composition of the source is small and will not be considered.

When the source subtends the maximum solid angle $2 \pi$ at the sample and is uniformly bright over its whole extent, the illumination on the sample is completely diffused. When the source subtends a relatively small solid angle at the sample, the illumination is characterized as unidirectional. To be more specific in this case, however, we state further that the solid angular extent $d \omega$ of the incident flux shall be so small that a further decrease will not alter appreciably either the angular distribution of the reflected flux or its ratio to the incident flux. If the illumination is neither completely diffused nor unidirectional, it will be spoken of simply as diffused.

The source for unidirectional illumination may be represented either as a "point" source of given intensity or as a small extended source of uniform brightness. The commonly used coil-filament incandescent lamp is, perhaps, a closer approximation, physically, to the small extended source than to the point source. In the following discussion it will be most convenient to express the illumination ${ }^{8}$ in the unidirectional case as $B \cos \theta d \omega$, where $B$ is the brightness of a small extended source subtending the small solid angle $d \omega$ at the sample and $\theta$ is the angle of incidence. To maintain a definite illumination, then, if $d \omega$ is decreased to meet the unidirectional specification, the brightness of the source must be increased correspondingly to compensate for the increase in distance or decrease in area accompanying the decrease in $d \omega$.

For the convenient expression of the total illumination on the sample, in the case of a large extended source, we may imagine the actual brightness distribution $B(\theta, \phi)$ replaced by a uniform distribution of constant value $B_{0}$ extending over a complete hemisphere surface (subtending a solid angle $2 \pi$ at the sample) and defined by the equation

$$
B_{0}=\frac{\int B(\theta, \phi) \cos \theta d \omega}{\int \cos \theta d \omega}
$$

in which the integrals are taken over the surface of the hemisphere. This may be expressed more simply, as

$$
\pi B_{0}=\int B(\theta, \phi) \cos \theta d \omega
$$

$B_{0}$ will be called the equivalent-hemisphere brightness of the source, and $\pi B_{0}$ is the total illumination on the sample. In the evaluation of the unidirectional illumination (see Section VI) it is also most con-

\footnotetext{
${ }^{8} \mathrm{~A}$ similar expression for the illumination from a point source would be $I \Omega$, where $I$ is the intensity of the source and $\Omega$ is the solid angle subtended at the source by unit area of the sample.
} 
venient for our purpose to express this illumination in terms of the equivalent-hemisphere brightness $B_{0}$. In this case equation (3) reduces simply to

$$
\pi B_{0}=B \cos \theta d \omega
$$

The equivalence of the uniform hemispherical source and the actual source for all types of illumination is only true, of course, with regard to the total illumination on the sample. They are not equivalent with regard to the angular distribution and total quantity of the reflected flux.

The total flux reflected by the sample may be represented, similarly, by $\pi B^{\prime}{ }_{0}$, where $B^{\prime}{ }_{0}$ is the average value of the brightness function $B^{\prime}\left(\theta^{\prime}, \phi^{\prime}\right)$, and defined by the equation

$$
\pi B^{\prime}{ }_{0}=\int B^{\prime}\left(\theta^{\prime}, \phi^{\prime}\right) \cos \theta^{\prime} d \omega^{\prime}
$$

$B^{\prime}{ }_{0}$ is obviously the brightness that the sample would have (observed in any direction) if it were a perfect diffusor ${ }^{9}$ reflecting the same total flux.

Taking, now, the observed brightness of the sample in a given direction $\left(\theta^{\prime}, \phi^{\prime}\right)$, multiplied by $\pi$, as a measure of the total reflected flux, we define the apparent ${ }^{10}$ reflectance (for the given direction of observation) as the ratio of $\pi B^{\prime}\left(\theta^{\prime}, \phi^{\prime}\right)$ to the total illumination on the sample. The group of apparent reflectances for various directions of observation $\left(\theta^{\prime}, \phi^{\prime}\right)$ will be denoted by the symbol $A\left(\theta^{\prime}, \phi^{\prime}\right)$, in which the manner of illumination is definite, but not explicitly specified. We have, then,

$$
A\left(\theta^{\prime}, \phi^{\prime}\right) \equiv \frac{\pi B^{\prime}\left(\theta^{\prime}, \phi^{\prime}\right)}{\pi B_{\mathrm{o}}}=\frac{B^{\prime}\left(\theta^{\prime}, \phi^{\prime}\right)}{B_{\mathrm{o}}}
$$

The apparent reflectance is, obviously, the reflectance which must be assigned to the sample, if, on the assumption that it is a perfect diffusor, it is to have the observed brightness. If the illumination on the sample is one lumen per square centimeter, the apparent reflectance is numerically equal to the observed brightness expressed in lamberts.

The definition of the reflectance given in equation (1) may now be rewritten, either by direct substitutions from equations (5) and (3), or by introducing the equivalent-hemisphere brightness $B_{0}$ of the

${ }^{9}$ A perfect diffusor is a sample obeying the cosine law of emission for any angular or spectral distribution of the incident flux. This assumes that the flux per unit solid angle in a given direction of reflection is directly proportional to the projected area of the element of surface; hence the brightness of the surface is constant for all angles of view. The perfect diffusor is a very convenient ideal reference "sample" for many purposes.

10 The use of the word apparent as well as the term reflectance was suggested by A. H. Taylor in the 1922 report (unpublished) of the Committee on Reflectometry of the Optical Society of America. 
source from (2) and the apparent reflectance $A\left(\theta^{\prime}, \phi^{\prime}\right)$ of the sample from (6). Thus:

$$
\begin{aligned}
R=\frac{B_{0}^{\prime}}{B_{0}} & =\frac{\int A\left(\theta^{\prime}, \phi^{\prime}\right) \cos \theta^{\prime} d \omega^{\prime}}{\int \cos \theta^{\prime} d \omega^{\prime}} \\
& =\frac{1}{\pi} \int A\left(\theta^{\prime}, \phi^{\prime}\right) \cos \theta^{\prime} d \omega^{\prime}
\end{aligned}
$$

in which the integrals are to be evaluated, as before, over the surface of a hemisphere (solid angle $2 \pi$ ). The expanded form of $\pi$ must be retained in the expression for $R$ when the approximate evaluation of the integrals (to be explained) is effected. The reflectance for any type of illumination is most conveniently expressed and calculated as a (weighted) mean value of the apparent reflectances for that illumination.

\begin{tabular}{|c|c|}
\hline Symbol & Interpretation \\
\hline$(\theta, \phi)$ & Brightness distribution over a finitely extended source, as viewed \\
\hline & Equivalent-hemisphere brightness of source. \\
\hline$B^{\prime}(\theta$ & $\begin{array}{l}\text { Brightness of sample viewed in various directions }\left(\theta^{\prime}, \phi^{\prime}\right) \text { for a } \\
\text { definite type of illumination not explicitly specified. }\end{array}$ \\
\hline$B^{\prime}$ & $\begin{array}{l}\text { Brightness the sample would have (viewed in any direction) if it } \\
\text { were a perfect diffusor reflecting the same total flux. }\end{array}$ \\
\hline & Reflectance. (Type of illumination not specified.) \\
\hline$A$ & $\begin{array}{l}\text { Group of apparent reflectances for various directions of observa- } \\
\text { tion }\left(\theta^{\prime}, \phi^{\prime}\right) \text { and for a definite type of illumination not explicitly } \\
\text { specified. }\end{array}$ \\
\hline $\begin{array}{l}\text { Subscripts, } \\
\quad \mathrm{D}, \mathrm{d}, \mathrm{U}, \theta \phi\end{array}$ & $\begin{array}{l}\text { Specify explicitly the type of illumination; that is, diffused (a), } \\
\text { completely diffused (D), unidirectional (U), and unidirectional } \\
\text { in the specified direction }(\theta, \phi) \text {, respectively. }\end{array}$ \\
\hline
\end{tabular}

TABLE 1.-Nomenclature

SOME ILLUSTRATIONS OF THE USE OF THE NOMENCLATURE ARE AS FOLLOWS

\begin{tabular}{c|c}
\hline$R_{\mathrm{U}}(\theta, \phi) \ldots \ldots$ & $\begin{array}{l}\text { Group of unidirectional reflectances for various directions of inci- } \\
\text { dence. } \\
\text { Group of apparent unidirectional reflectances for a particular direc- } \\
\text { tion of illumination }(\theta, \phi) \text { and various directions of observation. } \\
\text { Complete set of apparent unidirectional reflectances for all direc- } \\
\text { tions of incidence and observation. The first-named angles } \\
\text { always denote the direction of incidence; the last-named angles, } \\
\text { the direction of observation. }\end{array}$
\end{tabular}

Subscripts will be used to designate explicitly a specific type of illumination, as explained in Table 1. Thus, for completely diffused illumination, the reflectance is

$$
R_{\mathbf{D}}=\frac{\int A_{\mathbf{D}}\left(\theta^{\prime}, \phi^{\prime}\right) \cos \theta^{\prime} d \omega^{\prime}}{\int \operatorname{S} \cos \theta^{\prime} d \omega^{\prime}}
$$


The complete group of unidirectional reflectances for various directions of incidence are represented by

$$
R_{\mathrm{U}}(\theta, \phi)=\frac{\int A_{\mathrm{U}}\left(\theta, \phi ; \theta^{\prime}, \phi^{\prime}\right) \cos \theta^{\prime} d \omega^{\prime}}{\int \cos \theta^{\prime} d \omega^{\prime}}
$$

It is of interest to note at this point that the reflectance for any manner of diffused illumination may also be expressed as a mean value of the reflectances for unidirectional illumination. The group of unidirectional reflectances $R_{\mathrm{U}}(\theta, \phi)$ constitute a function given over the surface of an imaginary hemisphere with the sample at the center of its base. The diffuse reflectance $R_{\mathrm{d}}$ is then a mean value of the function $R_{\mathrm{U}}(\theta, \phi)$ averaged over the hemisphere, each unidirectional reflectance being weighted by the brightness of the source $B(\theta, \phi)$ in the corresponding direction and by the factor $\cos \theta d \omega$. These factors together give the portion of the total illumination of the sample which is received through an element of the hemispherical surface. Hence we may write

$$
R_{\mathrm{d}}=\frac{\int R_{\mathrm{U}}(\theta, \phi) B(\theta, \phi) \cos \theta d \omega}{\int \mathcal{S} B(\theta, \phi) \cos \theta d \omega}
$$

and for a uniform source (completely diffused illumination)

$$
R_{\mathrm{D}}=\frac{\int R_{\mathrm{U}}(\theta, \phi) \cos \theta d \omega}{\int \cos \theta d \omega}
$$

\section{RECIPROCAL RELATIONS}

As a consequence of a general law of reciprocity, first stated by Helmholtz, ${ }^{11}$ important reciprocal relations between certain groups of reflection coefficients may now be derived. A statement of the general law, as applied to the case here considered, is that the elementary pencils of Figure 1 are reversible with regard to both direction and angular flux density. ${ }^{12}$ Because the angular flux density in the direction of incidence is the same in both the direct and reciprocal cases, it follows from the law of reciprocity that the angular flux density in the direction of reflection is also the same in both cases, or

$$
B^{\prime}{ }_{\theta \phi}\left(\theta^{\prime}, \phi^{\prime}\right) \cos \theta^{\prime}=B_{\theta^{\prime} \phi^{\prime}}^{\prime}(\theta, \phi) \cos \theta
$$

\footnotetext{
${ }^{11}$ Helmholtz: Physiological Optics, 3d ed. 1909; translated by J. P. C. Southall and published by the Optical Society of America, 1924; Vol. I, p. 231. Vorlesungen über die Theorie der Wärme Herausgegeben von F. Richarz, Leipzig, J. A. Barth; 1903, p. 161. The loss in flux density which an infinitely narrow bundle of rays of definite wave length and state of polarization undergoes on its path through any medium by reflection, refraction, absorption, and scattering is exactly equal to the loss in flux density suffered by a bundle of the same wave length and polarization pursuing an exactly opposite path. Certain restrictions to the law were stated by Helmholtz, which have, however, no bearing on the present work.

${ }_{12}$ Angular flux density in a specified direction from a small emitting surface of brightness $B$ is equal to $B$ multiplied by the projected area of the surface in the direction considered.
} 
Multiplying both sides of the equation by $\pi / B d \omega$, we have

$$
\frac{\pi B^{\prime}{ }_{\theta \phi}\left(\theta^{\prime}, \phi^{\prime}\right)}{B \cos \theta d \omega}=\frac{\pi B^{\prime}{ }_{\theta^{\prime} \phi^{\prime}}(\theta, \phi)}{B \cos \theta^{\prime} d \omega}
$$

The denominators on each side of this equation express the illuminations of the sample in the direct and reciprocal cases, respectively. Using equation (4) and definition (6), it becomes

$$
A_{\theta \phi}\left(\theta^{\prime}, \phi^{\prime}\right)=A_{\theta^{\prime} \phi^{\prime}}(\theta, \phi)
$$

or, more generally expressed (Table 1 )

$$
A_{\mathrm{U}}\left(\theta, \phi ; \theta^{\prime}, \phi^{\prime}\right)=A_{\mathrm{U}}\left(\theta^{\prime}, \phi^{\prime} ; \theta, \phi\right)
$$

The apparent unidirectional reflectances for all directions of incidence and observation are thus seen to form a symmetrical function of the pairs of variables $\theta, \phi$ and $\phi^{\prime}, \theta^{\prime}$; a condition which (incidentally) must be satisfied by any theoretical or empirical formula used to represent the reflection characteristics of a sample.

By averaging the function $A_{\mathrm{U}}\left(\theta, \phi ; \theta^{\prime}, \phi^{\prime}\right)$ over a hemisphere (solid angle $2 \pi$ ), with respect to the pair of variables $\left(\theta^{\prime}, \phi^{\prime}\right)$, we obtain the reflectances for unidirectional illumination, as in equation (10). Averaging the same function again over the same limits with respect to the variables $(\theta, \phi)$, obviously yields the apparent reflectances for completely diffused illumination. As the pairs of variables $(\theta, \phi)$ and $\left(\theta^{\prime}, \phi^{\prime}\right)$ (defining directions of incidence and observation, respectively) are interchangeable by the general reciprocal relation, equation (14), we may write

$$
\frac{\int A_{\mathrm{U}}\left(\theta, \phi ; \theta^{\prime}, \phi^{\prime}\right) \cos \theta d \omega}{\int \cos \theta d \omega}=\frac{\int A_{\mathrm{U}}\left(\theta, \phi ; \theta^{\prime}, \phi^{\prime}\right) \cos \theta^{\prime} d \omega^{\prime}}{\int \cos \theta^{\prime} d \omega^{\prime}}
$$

when $\theta^{\prime}$ and $\phi^{\prime}$ on the left-hand side of the equation are equal to $\theta$ and $\phi$, respectively, on the right-hand side. Otherwise stated, we have, by definition

when

$$
\begin{gathered}
A_{\mathrm{D}}\left(\theta^{\prime}, \phi^{\prime}\right)=R_{\mathrm{U}}(\theta, \phi) \\
\left(\theta^{\prime}, \phi^{\prime}\right)=(\theta, \phi)
\end{gathered}
$$

Thus the apparent reflectance for completely diffused illumination is equal to the reflectance for unidirectional illumination, when the direction of observation in the first case is the same as the direction of incidence in the second case. In other words, they are both identical functions of the direction of observation $\left(\theta^{\prime}, \phi^{\prime}\right)$ and the direction of incidence $(\theta, \phi)$, respectively. 


\section{NEW METHOD IN REFLECTOMETRY}

To obtain the reflectance for unidirectional illumination, the necessary integration (with respect to direction) of the reflected flux, if not performed by some approximate integrating device (such as the Ulbricht sphere), must be done by actual measurements of the angular flux density of the reflected light in various directions from the sample and performing the operations indicated in equation (10). This generally requires a large number of observations and considerable computation.

Given the apparent reflectances for completely diffused illumination, however, the group of unidirectional reflectances are known at once (by equation (15)), and the reflectance for any type of illumination, ranging from unidirectional to completely diffused, may be readily computed by taking properly weighted mean values (using equations (11) or (12), which may be reduced to simpler form in the manner discussed later in Section VI).

An arrangement providing completely diffused illumination of known amount, with means for observing the brightness of the sample at any desired angle from the normal in one azimuth, is, then, an absolute reflectometer of a most general type, depending on no theory whatever of the action of an integrating device. Because of the symmetry of the illumination in this case brighiness measurements for other azimuths may be made (if desired) by rotation of the samole to different positions in its own plane.

Although the preceding sections contain an adequate proof of the above statements, it will be instructive, nevertheless, to apply the general theory to a particular case in order to bring out the essential features of the method and to illustrate clearly the actual integrating process involved. For these purposes the sources for completely diffused and unidirectional illumination will be constructed in a specified manner, so that a definitely known relation exists between the illuminations on the sample as received from each of the sources.

The first step in the demonstration of the method will be an application of the integral reciprocal relation, equation (15), to derive the relation that must exist in this case between the total reflected flux for the unidirectional illumination and the reflected flux for the completely diffused illumination. Let $B$ (fig. $2(a)$ ) represent the brightness of a small luminous area subtending a small solid angle $d \omega$ at the sample and satisfying the present requirements in a source for unidirectional illumination. The illumination on the sample is $B \cos \theta d \omega$. The reflected flux is distributed in an irregular manner, as indicated in the figure, and a measurement of its total 
quantity is desired. Let $F_{\theta \phi}$ be the total reflected flux per unit solid angular extent of the incident flux. Then

$$
R_{\mathrm{U}}(\theta, \phi)=\frac{F_{\theta_{\phi}} d \omega}{B \cos \theta d \omega}
$$

Let us now construct the source for completely diffused illumination by extension of the source for unidirectional illumination uniformly over the surface of a hemisphere, keeping its brightness constant (fig. $2(b)$ ). Let $f_{\mathbf{D}}\left(\theta^{\prime}, \phi^{\prime}\right)$ represent the angular flux density in the direction $\left(\theta^{\prime}, \phi^{\prime}\right)$ for the hemspherical source of uniform brightness $B$. The total illumination is $\pi B$ and the brightness of the sample, $B^{\prime}\left(\theta^{\prime}, \phi^{\prime}\right)$, viewed from direction $\left(\theta^{\prime}, \phi^{\prime}\right)$, is $f_{\mathbf{D}}\left(\theta^{\prime}, \phi^{\prime}\right) / \cos \theta^{\prime}$. Then

$$
A_{\mathrm{D}}\left(\theta^{\prime}, \phi^{\prime}\right)=\frac{\pi B^{\prime}{ }_{\mathrm{D}}\left(\theta^{\prime}, \phi^{\prime}\right)}{\pi B}=\frac{f_{\mathrm{D}}\left(\theta^{\prime}, \phi^{\prime}\right)}{B \cos \theta^{\prime}}
$$

and, by equation (15) with the above expression for $R_{\mathrm{U}}(\theta, \phi)$

when

$$
F_{\theta \phi}=f_{\mathrm{D}}\left(\theta^{\prime}, \phi^{\prime}\right)
$$

$$
(\theta, \phi)=\left(\theta^{\prime}, \phi^{\prime}\right)
$$

This equation is merely a restatement of the integral reciprocal relation, equation (15), and is valid only for the special arrangement of sources here considered.

We will now make use of the general reciprocity law, as first stated in the preceding section, to reestablish equation (16) in a manner chosen specifically to demonstrate the integration process involved. For this purpose a particular type of sample is employed, having no regularity in its body or surface structure which would cause the apparent reflectances for a given type of illumination to depend on the orientation of the sample in its own plane.

Let light be incident in the solid angle $d \omega$ (as in fig. $2(a)$ ) and a photometer arranged to observe the sample in a direction $\left(\theta^{\prime}, \phi^{\prime}\right)$ for which $\theta^{\prime}=\theta$ and $\phi^{\prime}=\phi+\pi$ (direction of regular spécular reflection). Let this photometer be constructed to measure all the light reflected in a solid angle $d \omega^{\prime}$-equal in all respects to $d \omega$. Imagine the sample now transformed into a perfect mirror without changing its reflectance. The photometer than measures the total quantity of light which was diffusely reflected from the sample in its original state. Keeping the position of the photometer and the reflectance of the transformed sample constant, let the mirror surface be slightly roughened and some body reflection added, if desired, so that the reflected flux is no longer wholly contained in the solid angle $d \omega^{\prime}$ but is spread out into a larger solid angle $\omega . '$ The photometer no longer measures the total reflected flux, but now reads a lower value. Let the line $M$ (fig. $2(a)$ ), in the plane of the paper, represent the direc- 
tion of specular reflection and let $N$ be some other direction of reflection not necessarily contained in the same plane. Arrange an additional source, equal in all respects to the first source, with line $N$ as the direction of incidence. In accordance with the general reciprocity law, the flux density of the reflected light in the direction $N$ due to the first source is the same as that in the direction $(\theta, \phi)$ due to the second source. The reflective properties of the sample chosen are such that we may now rotate the second source, with the incident and reflected beams, about the normal to the sample as an axis, without altering the flux densities or the angular relation between the beams, until the direction of the reflected beam under consideration coincides with the direction $M$ of the photometer.

Considering in the same manner all directions of reflection of the incident beam from. the first source, the result is a gradual extension of the original source (keeping its brightness constant), each incre-

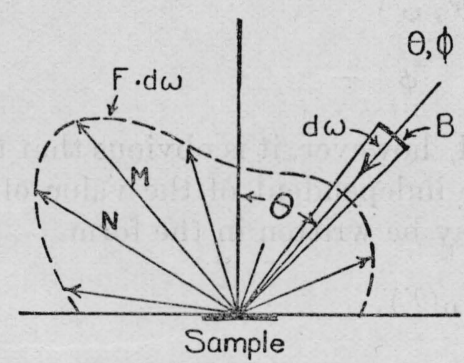

(a) - Unidirectional Illumination

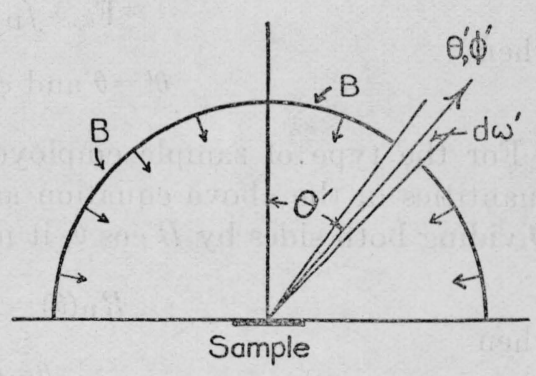

(b) Completely Diffused Illumination

FIG. 2.-Illustration of the application of the integral reciprocity law io reflectometry

ment of area increasing the photometer reading slightly, until the original reading for the perfect mirror sample is completely restored. When this condition is attained, the solid angular extent of the source (as subtended at the sample) corresponds in magnitude and shape to the solid angular extent $\omega^{\prime}$ of the reflected flux; in other words, for each direction of reflection $\left(\theta^{\prime}, \phi^{\prime}\right)$ there is now a corresponding direction of incidence $(\theta, \phi)$ such that $\theta=\theta^{\prime}$ and $\phi=\phi^{\prime}-\pi$. For every pencil of rays scattered out of the original solid angle $d \omega^{\prime}$ by the altered mirror sample there is now an equal pencil of rays scattered into the photometer from a different part of the extended source.

The substituted mirror sample may be continuously modified, introducing body reflection as desired, until its reflective properties are exactly the same as the original sample, the reflectance of which is required. The source is simultaneously extended until it subtends if necessary a complete hemisphere at the sample. During these changes the reading of the photometer remains constant and measures the total reflected flux for the original unidirectional illumination. 
The integration process involved in this experiment is evident. Instead of adding direct measurements of the angular flux density in the large number of directions from the sample (using equation (10)) or inventing a device to gather up automatically a known portion of the irregularly reflected flux and project it in one direction into a photometer, we may keep the photometer position fixed and effectively move the source about-covering all angles of incidence and adding the (unweighted) readings of the photometer for the different positions of the source. As the integrating sphere is used to obtain a direct measurement of the total reflected flux, so here the uniform hemispherical source and the reciprocity law are combined to obtain the same result by a single measurement in one direction from the sample.

The result may be expressed in the terms used above. We have, then,

when

$$
\mathrm{F}_{\theta_{\phi}}=f_{\mathbf{D}}\left(\theta^{\prime}, \phi^{\prime}\right)
$$

$$
\theta^{\prime}=\theta \text { and } \phi^{\prime}=\phi+\pi
$$

For the type of sample employed, however, it is obvious that the quantities in the above equation are independent of the value of $\phi$. Dividing both sides by $B \cos \theta$, it may be written in the form

when

$$
R_{\mathbf{U}}(\theta)=A_{\mathbf{D}}\left(\theta^{\prime}\right)
$$

$$
\theta=\theta^{\prime}
$$

In the more general case (equations (15) and (16)), where no restrictions are made on the type of sample considered, it is necessary that the direction of observation for completely diffused illumination (uniform hemispherical source) be in the same azimuth as the direction of incidence for the unidirectional illumination.

The theories of the instruments which have been devised for the direct integration of the reflected flux are based upon assumptions which are not always satisfactorily realized in practice (see Sections $\mathrm{V}$ and VIII). Moreover, it is usually difficult to obtain a sufficient illumination on the sample from one direction to enable the use of a spectrophotometer for the measurement of absolute spectral reflectances.

The method here described has its theoretical basis in the reciprocity law, the truth of which can not be doubted. Its general applicability to the measurement of all the reflection coefficients commends its use. It has the further advantage that the source, being extended over a hemisphere, may be readily constructed to bring a large number of lamps into use, thus obtaining a sufficient illumination on the sample for precise spectrophotometric measurements throughout the whole visible spectral range. 
An equipment of this kind and its method of use (without the spectrophotometer) is described in detail in Section VI. The experimental errors likely to result from an incomplete diffusion of the illumination, in the evaluation of the constant of the instrument $1 / B_{0}$, and in the measurement of sample brightness are there fully discussed.

Inasmuch as the quantities previously defined and the reciprocal relations derived may all be stated in an analogous manner for the transmitted flux, the method here described is equally applicable to the measurement of the analogous transmission coefficients.

\section{THEORY OF THE SPHERE REFLECTOMETER}

\section{METHODS OF SHARP AND LITTLE AND OF KARRER}

In these methods the sample forms a relatively small portion of the wall of a hollow sphere, the interior surface of which is coated with a good diffusing material such as magnesium oxide. A narrow beam of light, projected in to the sphere, illuminates directly a small spot on the wall, the first reflected light from which is screened from the sample. Assuming the material of the sphere wall to be a perfect diffusor, it follows from the geometry of the sphere that any one element of the wall illuminates all other elements equally. ${ }^{13}$ Multiple reflections within the sphere increase this illumination many times. If, in addition to being a perfect diffusor, the reflectance of the sphere wall is the same over all its parts, each element of the wall receives the same quantity of light from every other element (excluding the directly illuminated spot). The area of the sphere wall occupied by the sample is assumed to be so small that its presence or absence does not appreciably affect the illumination of the remaining part. Under these conditions the brightness $B$ of the sphere is constant over all its parts. It serves as an extended source of uniform brightness subtending a solid angle of $2 \pi$ at the sample. The illumination on the sample is completely diffused and of total amount $\pi B$ (by equation (3)).

In the use of the sphere the sample is observed in a fixed direction from the normal to the surface and its brightness $B^{\prime}$ compared with that of the adjacent sphere wall; that is, with that of the source. Assuming the aforesaid conditions to be exactly realized in the construction of the sphere, the quantity measured is the apparent reflectance for completely diffused illumination and a particular direction of observation. This quantity is always equal to the reflectance $R_{\mathrm{D}}$ (for the same illumination) for at least one direction of observation (by equation (7)), but not for all directions of observation unless the reflective properties of the sample are such that the brightness

${ }^{13}$ Sumpner, Phil. Mag., 35, p. 81; 1893. Ulbricht, Electrotechn. Zs., 21, p. 595; 1900; 26, p. 152; 1905. 
(under completely diffused illumination) is independent of the angle of view. When this condition is satisfied, the total reflected flux is $\pi B^{\prime}$ (equation (5)), and we have

$$
R_{\mathrm{D}}=\frac{B^{\prime}}{B}
$$

Because this sphere method theoretically gives the reflectance for the extreme case of the perfect diffusor (for which the brightness is, by definition, independent of the angle of view), and for purely specular reflection, as in the case of a metal mirror (for which the unidirectional reflectance is nearly independent of the angle of incidence), it has been assumed that the correct value of the reflectance $R_{\mathrm{D}}$ would be obtained for the case of any gradation of mixed specular and diffuse reflection between these extremes. ${ }^{14}$

The incorrectness of this assumption was first pointed out by Walsh, ${ }^{15}$ who used an empirical expression to represent the reflection characteristics of a sample and showed by his calculations the possibility of large differences between the apparent reflectance, as measured with the sphere, and the true reflectance. Further results of the computations by Walsh are not substantiated, however, in the present investigation. (See Section VII.)

It is obvious that the sphere, as here used, has no integrating action whatever on the reflected flux. It serves merely as a means of obtaining a completely diffused illumination on the sample. The angular flux density is measured in one direction of reflection only (usually nearly normal to sample), and it is only when this direction is properly chosen for each sample (see Section VIII) that the reflectance $R_{\mathrm{D}}$ is obtained.

In the light of the reciprocity law this use of the Ulbricht sphere may better be regarded as a special case of the general method of reflectometry discussed in the preceding section. Then the quantity measured should be the reflectance for unidirectional illumination at a specified angle of incidence, and the diffusing properties of the sample itself need not be considered in the method.

\section{THE TAYLOR REFLECTOMETER}

In this instrument the sample likewise forms a small portion of the wall of a hollow sphere, the interior surface of which is covered with a good diffusing material of high reflectance. A narrow beam of light is projected into the sphere, either on the sample at a specified angle from the normal or on another portion of the sphere wall, as desired. A photometer compares the brightness of a third portion. of the wall (shaded from the sample by an opaque screen) under two conditions: (1) When the projected beam is incident on the sample, 
and (2) when it is incident on the sphere wall. The brightness of the observed spot is due in the first case to light reflected once from the sample and then multiply reflected from the sphere wall. The brightness in the second case is due to the light multiply reflected from the sphere wall only. The ratio of these two brightnesses gives, under certain restrictions, the reflectance of the sample.

The area occupied by the sample must be sufficiently small to be ineffective in altering the observed ratio of brightnesses. The sphere wall must also be a perfect diffusor with uniform reflectance over all its parts. Under these conditions the irregularly reflected flux from the sample is accurately integrated with respect to all directions of reflection, and a definitely known portion of the total reflected flux is projected into the photometer.

Consider the flux reflected from the sample in the direction $\left(\theta^{\prime}, \phi^{\prime}\right)$ and falling on a small area $a$ of the sphere wall. This area subtends a solid angle $d \omega$ at the sample, and if $f\left(\theta^{\prime}, \phi^{\prime}\right)$ be the angular flux density in the direction $\left(\theta^{\prime}, \phi^{\prime}\right)$, the flux incident on the element $a$ is $f\left(\theta^{\prime}, \phi^{\prime}\right) d \omega$. The reflected flux is $R^{\prime} f\left(\theta^{\prime}, \phi^{\prime}\right) d \omega$, where $R^{\prime}$ is the reflectance of the sphere wall.

The element $a$ illuminates all other elements of the sphere wall equally; hence the illumination of the observed spot is

$$
\frac{R^{\prime} f\left(\theta^{\prime}, \phi^{\prime}\right) d \omega}{A}
$$

where $A$ is the total area of the sphere wall. Multiple reflections in the sphere now increase this illumination by the factor ${ }^{16} \frac{1}{1-R^{\prime}}$. Hence, the total illumination of the observed spot, due to the portion of the total reflected flux under consideration, is

$$
\frac{R^{\prime}}{A\left(1-R^{\prime}\right)} f\left(\theta^{\prime}, \phi^{\prime}\right) d \omega
$$

It is independent of the position of the element $a$ but is proportional to the illumination of the element received from the sample.

The observed spot always sends a definite fraction of the total incident flux into the photometer, regardless of the angular distribution of the incident flux. Its brightness $d B_{\mathrm{s}}$ is $R^{\prime} / \pi$ times its total

\footnotetext{
${ }_{16}$ See Karrer, B. S. Sci. Paper No. 415, August, 1921, p. 215; also Taylor and Rosa, B. S. Sci. Paper No. 447; August, 1922, p. 307.

If $F$ be the total flux projected (or emitted) into an inclosure with perfectly diffusing wall (such as the hollow sphere), the average total illumination $E$ of the wall is

$$
E=\frac{F}{A\left(1-R^{\prime}\right)}
$$

where $A$ is the total area of the wall and $R^{\prime}$ its average reflectance. The average direct illumination $E_{\mathrm{d}}$ is $F / A$, hence

$$
E=\frac{E_{\mathrm{d}}}{1-R^{\prime}}
$$
}


illumination. Hence,

$$
d B_{\mathrm{s}}=\frac{R^{\prime 2}}{\pi A\left(1-R^{\prime}\right)} f\left(\theta^{\prime}, \phi^{\prime}\right) d \omega
$$

Considering, now, in the same manner the flux reflected in all directions from the sample, the total brightness of the observed spot is

$$
B_{\mathrm{s}}=\frac{R^{\prime 2}}{\pi A\left(1-R^{\prime}\right)} \int f\left(\theta^{\prime}, \phi^{\prime}\right) d \omega=k F^{\prime}
$$

where the integral is taken over the solid angle $2 \pi, F^{\prime}$ is the total reflected flux, and $k$ is the constant factor outside the integral sign.

Let $F$ be the total flux projected into the sphere on to the sample. To evaluate $F$ it is projected on to the sphere wall and, by exactly the same procedure as before, we find the brightness $B_{\mathrm{w}}$ of the observed spot to be in this case

$$
B_{\mathrm{w}}=k F
$$

Hence, the reflectance $R$ of the sample is

$$
R=\frac{F^{\prime}}{F}=\frac{B_{\mathrm{s}}}{B_{\mathrm{w}}}
$$

In this method it is obvious that no assumption is made in regard to the diffusing properties of the sample. The instrument theoretically measures the reflectance for unidirectional illumination at a specified angle of incidence.

\section{MEASUREMENT OF APPARENT REFLECTANCE}

\section{DESCRIPTION OF SAMPLES}

The samples used include various kinds of material selected with respect to two widely variable characteristics, namely, the degree of departure from the perfect diffusor (extreme case represented by a mirror) and the reflectance. These characteristics are, in general, functions of the spectral composition of the incident light and its angular distribution. The purposes of this investigation have been served, however, by limiting the choice of samples to those approximately neutral in color; so that the spectral composition of the incident and reflected light need not be taken into consideration, and the complications of heterochromatic photometry, or of spectrophotometry, are thereby avoided. Likewise, samples having a regular body structure or surface texture (such as a distinct weave or corrugation) are not included, as they complicate the measurements by introducing another variable (an azimuth angle) and are not of particular interest here.

The samples are listed in Table 2, with the reflectances and other data. The paper and cloth samples were mounted on aluminum 
plates (using dry-mounting tissue) to insure flatness of surface and permanence of form. Further description of the samples, indicative of their departure from the perfect diffusor, is afforded by the curves (io be described) in Figures 5, 6, and 7 .

\section{APPARATUS AND METHOD FOR DIFFUSED ILLUMINATION}

In this section equipment is described with which the apparent reflectances for diffused illumination may be measured and the reflectance computed (by equation (9)). The essential parts of the apparatus are shown in Figure 3 . The sample $S$ is supported from below against the rectangular opening $O$ in the center of and parallel to the base of a milk-glass hemisphere $M$, the outer surface of which is illuminated by 104 small tungsten filament lamps mounted on the hemispherical shell $A l$, which is concentric with the milk-glass hemisphere. The interior surface of the aluminum hemisphere and the whole base is covered with a deposit of magnesium oxide. The lamps are arranged on six parallels of latitude, equally spaced from the first ring of four lamps (about the pole) to the base. The spacing of the lamps on each ring is decreased progressively toward the bottom to partly compensate for the decreased direct illumination of the lower part of the milk-glass hemisphere. The current in each ring of lamps is separately adjustable.

To locate points on the hemisphere and specify directions from the normal to the sample (polar axis of hemisphere), let $\theta$ (as in fig. 1) be the angle from the polar axis and $\phi$ the azimuth angle measured (clockwise from above) from the meridian plane through $N$. The sample is under diffused illumination from the hemispherical source and may be viewed at angles of $0,12.0,25.5,39.0,52.5,66.0$, and $78.5^{\circ}$, respectively, through seven small holes cut through the milkglass hemisphere along the $180^{\circ}$ meridian and corresponding holes in the outer hemisphere. The tube $T$, with small aperture at $a$, extends from each hole in the outer hemisphere to within a few centimeters of the milk glass and serves to prevent the entrance into the optical observing system of the direct light from the hemisphere. These tubes are blackened on the inside and coated on the outside with magnesium oxide. The arm $A$, supporting prisms $P_{3}$, $P_{2}, P_{1}$, and lenses $L_{2}, L_{1}$, may be rotated about a horizontal axis through the center of the hollow bearing $B$ and held fixed in any position by a clamp $C$.

Brightness measurements at any of the above angles of view are made as follows: With the sample in place at $S$ and the arm clamped in position at the desired angle, a beam of light from the sample is directed by the prisms and lenses along the axis of rotation of the arm and serves to illuminate one-half of the biprism photometric field $B-L$. This field is viewed through the aperture $E$. The other 

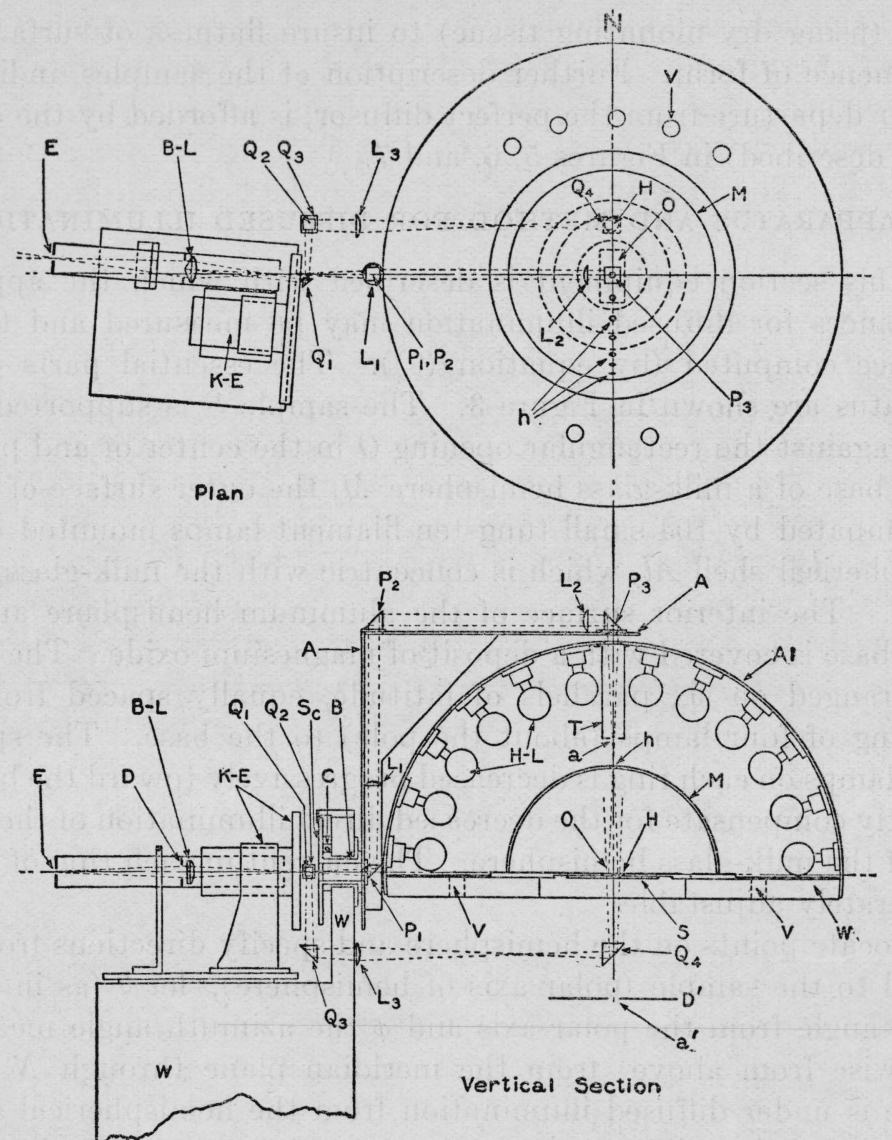

Fig. 3.-Apparatus for the measurement of apparent reflectances under completely diffused illumination

Lower diagram is vertical section through polar axis of hemisphere. Upper diagram is view with outer hemisphere and other supports removed.

$M$, milk-glass hemisphere, 9 inches in diameter, held in position on aluminum base plate. $A l$, aluminum hemisphere, 2 feet in diameter (built up in sectors), supporting lamps and serving as a reflector. $H-L$, hemisphere lamps; 9 volt, 27 c. p. automobile headlight lamps, 104 in all; filaments approximately $13 \mathrm{~cm}$ from milk-glass hemisphere. $S$, sample held in position by clamp (not shown). $O$, opening in base plate for sample, size 6 by $2 \mathrm{~cm}$. Surface of sample is $1 \mathrm{~mm}$ below inner surface of hemisphere base plate. $h$, holes in milk-glass hemisphere (each 6 $\mathrm{mm}$ diameter) through which sample is viewed at different angles. $P_{1}, L_{1}, P_{2}$, $P_{3}, L_{2}$, prisms and lenses of "sample" beam. $Q_{1}, Q_{2}, Q_{3}, L_{3}$, prisms and lenses of comparison beam. A, rotating arm supporting prisms and lenses of sample beam. $B$, hollow bearing for rotating arm. $C$, clamp to hold arm $A$ in desired position. $S c$, circular scale giving position of arm. $K-E$, Keuffel and Esser variable sectored disk photometer. $B-L$, biprism and lens forming photometric field. $H$, hole in base for comparison beam. T, tube with small aperture $a$ (one tube for each angle of view) to prevent entrance of direct light from hemisphere into optical observing system. $D^{\prime}$, movable opaque screen with small aperture, $a^{\prime}$, serving same purpose as tube $T$ in measurements of hemisphere brightness. $W$, wood supports. $V$, ventilating holes. A forced ventilation was necessary. This is not shown in diagram. 
half of the field is illuminated by a beam of light proceeding from a spot on the interior surface of the milk-glass hemisphere (at $\phi=0$, $\theta=35^{\circ}$ ) through a hole $H$ in the base and thence by way of fixed prisms and lenses $Q_{4}, L_{3}, Q_{3}, Q_{2}, Q_{1}$ to the biprism and lens combination $B-L$. The brightness of this half of the field can be controlled by means of a Keuffel and Esser variable sectored disk photometer. ${ }^{17}$

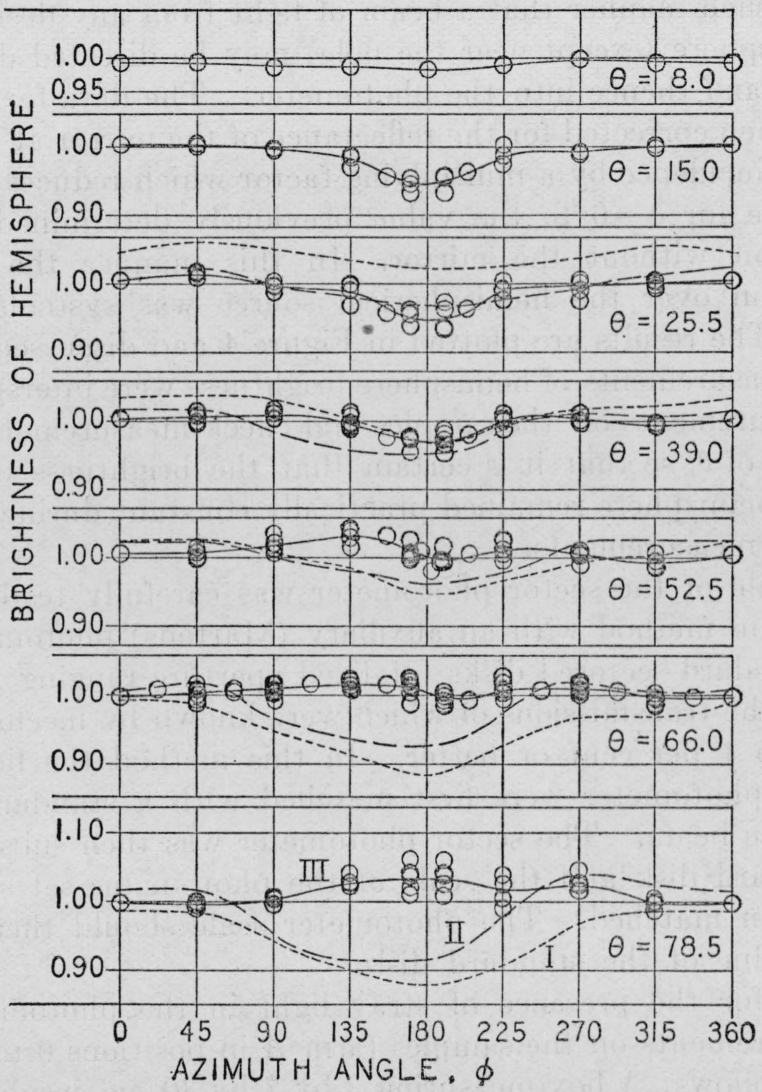

FiG. 4.-Brightness distribution over hemispherical source Dotted lines=distribution $\mathrm{I}$. Dash lines=distribution II. Full lines= distribution III.

By rotating the arm $A$ from 90 to $180^{\circ}$, the brightness distribution along the zero meridian of the hemisphere $\left(\phi=0 ; \theta=0\right.$ to $\left.90^{\circ}\right)$ may (with the sample removed) be measured directly. With the observing arm at $145^{\circ}$ the two beams of light proceed from the same spot on the wall; hence the brightness of this spot is taken as the unit in which all other brightness measurements, on the samples and source, are expressed. Photometric readings with the arm in this position varied, however, from time to time, due mainly to the accumulation

${ }_{17}$ C. W. Keuffel, J. Opt. Soc. Am. and Rev. Sci. Inst., 11, p. 403; October, 1925. $2284^{\circ}-28-4$ 
of dust on the exposed surfaces of the prisms and lenses. Consequently, these reference readings were always taken immediately before and after each set of readings on a sample (or the source).

To measure the brightness distribution over the remainder of the hemisphere, the arm $A$ is clamped in position at $180^{\circ}$ and a plane silvered-glass mirror mounted at the center of the base of the hemisphere in such manner that a beam of light from any desired part of the hemisphere (except near the pole) may be directed down to the prism $P_{3}$ and thence into the photometer. The data for any value of $\theta$ are then corrected for the reflectance of the mirror at the various angles of incidence by a multiplying factor which reduces the brightness value for $\phi=0$ to the value previously determined by direct observation without the mirror. In this manner the brightness distribution over the hemispherical source was systematically explored. The results are plotted in Figure 4 and discussed later.

The measurements of hemisphere brightness were interspersed with the measurements on the samples and check measurements made at all values of $\theta$, so that it is certain that the brightness distribution over the hemisphere remained practically constant during the course of all the measurements.

The scale of the sector photometer was carefully tested, using a substitution method with an auxiliary (Martens) photometer and a set of standard sectored disks (of fixed aperture ranging from 0.015 to 0.80 ), the transmissions of which were known by mechanical calibration to 1 per cent or better. In this method the fields of the auxiliary photometer were first matched with a standard sectored disk in one beam. The sector photometer was then substituted for the standard disk and the scale of the photometer set so that the fields again matched. The photometer scale should then read the known value of the standard disk.

A test for the presence of stray light in the photometric field, for measurements on the samples ( $\operatorname{arm} A$ in positions 0 to $90^{\circ}$ ), was made as follows: A box measuring 3 by 7 by $30 \mathrm{~cm}$ inside was lined on the two 7 by $30 \mathrm{~cm}$ sides and bottom with black velvet and the other two sides covered with strips of silvered-glass mirror. This box was mounted below the base of the hemisphere with its long axis coincident with the polar axis of the hemisphere and the mirror sides perpendicular to the zero meridian plane. An aperture in the top of the box coincided with the sample aperture at $O$ (fig. 3 ). The action of the mirrors permitted light to be taken only from the black bottom surface of the box for each position of the arm $A$, and the box thus served as a "sample" of practically zero refiectance. The photometer readings with this box in place were practically zero for each position of $A$, hence the stray light present was entirely negligible. For measurements of hemisphere brightness (arm $A$ in posi- 
tions 90 to $180^{\circ}$ ) a diaphragm $D^{\prime}$ was inserted in the focal plane of the lens $L_{2}$ with small aperture $a^{\prime}$ just large enough to allow the beam to pass through. This corresponds to the aperture $a$ and prevents the entrance into the optical system of extraneous light from the hemisphere.

The evaluation of the illumination on the sample from the brightness measurements over the hemispherical source is discussed in the following section. Knowing the illumination, then, the apparent reflectances are obtained from the brightnesses of the sample (by equation (6)) for certain angles of observation in one azimuth. If the brightness distribution over the hemisphere is sufficiently uniform so that the illumination of the sample may be regarded for practical purposes as completely diffused, the apparent reflectances for any other azimuth may then be obtained if desired, by rotation of the sample to different positions in its own plane and repeating the brightness" measurements on the sample for each new position. In this way a complete group of apparent reflectances may be obtained and the reflectance for completely diffused illumination computed (by equation (9)). The samples were chosen, however, to avoid the necessity of observations in all azimuths; and they all gave the same brightness under the illumination from the hemisphere when turned through $90^{\circ}$. Hence, the apparent reflectances of these samples for completely diffused illumination may be regarded as functions of $\theta^{\prime}$ alone.

The data are all given in Figure 5 and discussed later. The effect of the small departure of the illumination from complete diffusion will be shown to be of no practical importance.

\section{BRIGHTNESS DISTRIBUTION OVER HEMISPHERICAL SOURCE}

Three different brightness distributions have been used, each corresponding to a different adjustment of the currents in the lamps and their relative distances from the milk glass. The thickness of the milk-glass hemisphere varied from $11 / 2$ to $3 \mathrm{~mm}$ (approximately) over its different parts, causing corresponding variations in the brightness of the inner surface due to its variable transmission. The decreased transmission of the direct light from the lamps by the thicker portions of the hemisphere is partly compensated, however, by a greater reflection by these thicker parts of the light incident on the inner surface. The regions of low brightness in Distributions I and II are due primarily to the greater thickness. This was compensated in Distribution III by moving the lamps closer to the milk glass and by readjustment of the currents.

The data for Distribution III are all plotted as circles in Figure 4. Each single circle at $\phi=0$ is the mean of several points determined for this azimuth. The dip in the curves at $\phi=180^{\circ}(\theta=15.0,25.5$, 
and $39.0^{\circ}$ ) is not due to the presence of the holes in the hemisphere but to an appreciable shading of these regions of the hemisphere by the tubes $T$ (fig. 3). The dip is not shown for higher values of $\theta$ because of its small angular extent. With the exception of these small regions, the brightness distribution over the hemisphere (for Distribution III) is constant to within \pm 4 per cent of the average brightness and is constant to within \pm 2 per cent over 90 per cent of the whole hemisphere. The brightness along the zero meridian is constant to within \pm 1 per cent. Measurements were also made for values of $\theta$ equal to 32,45 , and $59^{\circ}$. These data are not shown in Figure 4 but were used in the calculation of the total illumination.

The equivalent-hemisphere brightness of the source is computed from these data by equation (2). Writing $d \omega=\sin \theta d \theta d \phi$ and evaluating the integrals over the hemisphere, we have

$$
\begin{aligned}
B_{0} & =\frac{\int_{0}^{2 \pi} \int_{0}^{\pi / 2} B(\theta, \phi) \cos \theta \sin \theta d \theta d \phi}{\int_{0}^{2 \pi} \int_{0}^{\pi / 2} \cos \theta \sin \theta d \theta d \phi} \\
& =\frac{\pi \int_{0}^{\pi / 2} \sin 2 \theta d \theta \frac{1}{2 \pi} \int_{0}^{2 \pi} B(\theta, \phi) d \theta}{\pi \int_{0}^{\pi / 2} \sin 2 \theta d \theta} \\
& =\int_{0}^{\pi / 2} \bar{B}(\theta) K(\theta) d \theta \\
& =\sum_{0}^{\pi / 2} \bar{B}(\theta) K(\theta), \text { approximately }
\end{aligned}
$$

where $\bar{B}(\theta)$ is the mean value of $B(\theta, \phi)$, averaged with respect to $\phi$, and $K(\theta)$ is a set of weights summing to unity.

$$
K(\theta)=\frac{\sin 2 \theta}{\int_{0}^{\pi / 2} \sin 2 \theta d \theta}=\frac{\sin 2 \theta}{\sum_{0}^{\pi / 2} \sin 2 \theta} \text {, approximately }
$$

A curve was drawn showing $\bar{B}(\theta)$ as a function of $\theta$, and values read from this curve at intervals of $5^{\circ}$ were multiplied by $K(\theta)$, computed for the same intervals. The value of $B_{0}$ obtained from the summation was 0.999 , for the brightness Distribution III.

The brightness Distributions I and II, represented by the dotted and dashed lines, respectively, in Figure 4, are the observed brightnesses in each case multiplied by the proper factors to give the same equivalent-hemisphere brightness as given by Distribution III. Thus, all three distributions (as represented in fig. 4) give the same total illumination on the sample. 


\section{METHOD FOR UNIDIRECTIONAL ILLUMINATION}

The apparatus of Figure 3 was designed so that the hemispherical source could readily be removed. The sample was then mounted at 0 and a 400-watt concentrated filament lamp placed at a distance of $50 \mathrm{~cm}$ from $O$, in the vertical plane through $N$ and $O$, and including the normal to the sample. A block of magnesium carbonate, placed in the position of $Q_{4}$, received light from the same lamp and served as a comparison source. This arrangement eliminates any effect on the photometer readings caused by variations in the intensity of the lamp.

The sample could be orientated to receive a beam of light (uncollimated) at any desired angle of incidence and the brightness of the sample observed at various angles of reflection in the plane of incidence. These brightnesses were all measured relative to the brightness of a porcelain plate, which was substituted for the sample and observed (by reflected light) at an angle of $50^{\circ}$ for normal illumination. The brightness of this plate served as a temporary unit in which all brightness measurements on the sample were expressed.

Readings on the porcelain plate were always taken immediately before and after each set of readings on a sample, so that any steady shifting of the photometer readings (due mainly to the gradual accumulation of dust on the optical surfaces) had no effect on the brightness measurements.

To obtain the apparent reflectances, it is now necessary to evaluate the illumination on the sample or, otherwise stated, to determine the equivalent-hemisphere brightness of the source expressed in the same unit of brightness used in the measurements on the samples. For this purpose a transmission standard ${ }^{18}$ was used. This consisted simply of a plate of milk glass of known apparent transmission ${ }^{19}$ for normal observation on one side and unidirectional normal illumination on the opposite side. The transmission standard was substituted for the porcelain plate and its brightness by transmitted light (viewed normal to the surface) measured relative to the brightness of the porcelain plate (unit brightness). The illumination in both cases was normal to the surfaces. Calling this relative brightness $B^{\prime \prime}$, the equivalent-hemisphere brightness $B_{0}$ is determined from the definition:

$$
\text { Apparent transmission }=\frac{B^{\prime \prime}}{B_{\mathrm{o}}}
$$

The apparent reflectances may now be obtained by equation (6). In the case of unidirectional normal illumination they can be meas-

${ }_{18}$ Loaned to the author by Bureau of Standards, division 1, section 5 (photometry). The calibration of this standard consisted in a determination of the brightness in lamberts of one side of the plate (observed normal to the surface) for a known illumination in lumens (incident normally on the opposite side).

10 A pparant transmission is analogous to apparent reflectance and defined in like manner as the brightness (by transmitted light) of the sample multiplied by $\pi$ and divided by the total illumination. 
ured for all azimuths (if desired) by rotation of the sample in its own plane. For all the samples studied, however, the apparent reflectances in the plane of observation were found to be practically independent of the azimuthal orientation of the sample. Hence, the brightness and apparent reflectance for this type of illumination may be regarded (as in the case of completely diffused illumination) as functions of $\theta^{\prime}$ alone. The data are shown in Figure 6 and are sufficient for the calculation of the reflectances.

To calculate the reflectance for any unidirectional illumination other than normal, the apparent reflectances for all azimuths of observation would be required. Complete data for this purpose could not be obtained with the above equipment. The apparent reflectances for $45^{\circ}$ illumination and angles of observation in the plane of incidence are given in Figure 7.

\section{CALCULATION OF REFLECTANCE}

Owing to the choice of samples and the symmetry of the illumination, it has been shown that the apparent reflectances for both completely diffused illumination and unidirectional normal illumination (figs. 5 and 6 , respectively) are independent of the azimuth angle $\phi^{\prime}$ of the direction of observation and are functions of $\theta^{\prime}$ alone. Hence equations (9) and (10) for the reflectances are simplified, and after evaluating $d \omega$, we have from (9)

and from (10)

$$
\begin{gathered}
R_{\mathrm{D}}=\frac{\int_{0}^{\pi / 2} A_{\mathrm{D}}\left(\theta^{\prime}\right) \sin 2 \theta^{\prime} d \theta^{\prime}}{\int_{0}^{\pi / 2} \sin 2 \theta^{\prime} d \theta^{\prime}} \\
=\sum_{0}^{\pi / 2} A_{\mathbf{D}}\left(\theta^{\prime}\right) K\left(\theta^{\prime}\right), \text { approximately }
\end{gathered}
$$

$$
\begin{gathered}
R_{\mathrm{U}}(0)=\frac{\int_{0}^{\pi / 2} A_{\mathrm{U}}\left(0 ; \theta^{\prime}\right) \sin 2 \theta^{\prime} d \theta^{\prime}}{\int_{0}^{\pi / 2} \sin 2 \theta^{\prime} d \theta^{\prime}} \\
=\sum_{0}^{\pi / 2} A_{\mathrm{U}}\left(0 ; \theta^{\prime}\right) K\left(\theta^{\prime}\right), \text { approximately }
\end{gathered}
$$

where $K\left(\theta^{\prime}\right)$ is defined by equation (18).

Values read from the curves of Figures 5 and 6 at $5^{\circ}$ intervals of $\theta^{\prime}$ were multiplied by $K\left(\theta^{\prime}\right)$ for the same intervals and the reflectance obtained from the summation. The computed reflectances for both types of symmetrical illumination are given in columns 2 and 13 of Table 2 . 


\begin{tabular}{|c|c|c|c|c|c|c|c|c|c|c|c|c|c|c|}
\hline \multirow{5}{*}{ Sample } & \multicolumn{9}{|c|}{ Completely diffused illumination } & \multicolumn{5}{|c|}{ Unidirectional illumination } \\
\hline & \multicolumn{5}{|c|}{ Hemispherical source; equipment of Figure 3} & \multicolumn{4}{|c|}{$\begin{array}{l}\text { Results with sphere reflectometer } \\
\text { (equipment of Figure 8; method es- } \\
\text { sentially same as proposed by Sharp } \\
\text { and Little and by Karrer }\end{array}$} & \multicolumn{3}{|c|}{$\begin{array}{l}\text { Equipment of Figure 3, modi- } \\
\text { fied for unidirectional illu- } \\
\text { mination }\end{array}$} & \multicolumn{2}{|c|}{$\begin{array}{c}\text { Measurements } \\
\text { with Taylor sphere } \\
\text { reflectometer }\end{array}$} \\
\hline & \multirow{3}{*}{$\begin{array}{c}\text { Reflect- } \\
\text { ance } \\
R_{\mathrm{D}}\end{array}$} & \multirow{2}{*}{\multicolumn{4}{|c|}{$\begin{array}{l}\text { Apparent reflectance } A_{\mathrm{D}}\left(\theta^{\prime}\right) \text { for obser- } \\
\text { vation angles of- }\end{array}$}} & \multirow{3}{*}{\begin{tabular}{|c|} 
Apparent \\
reflect- \\
ance \\
$A \mathrm{D}(7)$ \\
compare \\
with \\
columns \\
3 and 13 \\
and
\end{tabular}} & \multirow{2}{*}{\multicolumn{3}{|c|}{$\begin{array}{l}\text { Errors in measurement of } \\
\text { refiectance, resulting from } \\
\text { assumption that apparent } \\
\text { reflectance equals true re- } \\
\text { flectance for certain angles } \\
\text { of observation; computed } \\
\text { from columns } 2,3,5,6 \text {, for } \\
\text { observation angles of- }\end{array}$}} & \multirow{3}{*}{\begin{tabular}{|c|}
$45^{\circ}$ inci- \\
dence \\
\\
\\
Apparent \\
relect- \\
ance \\
$A_{\mathrm{U}}(45 ; 0)$
\end{tabular}} & \multicolumn{2}{|c|}{ Normal incidence } & \multirow{3}{*}{\begin{tabular}{|c|}
$30^{\circ}$ inci- \\
dence \\
Refiect- \\
ance, \\
Munsell \\
instru- \\
ment \\
$R_{\mathrm{U}}(00)$ \\
compare \\
with col- \\
umn 4
\end{tabular}} & \multirow{3}{*}{\begin{tabular}{|c|}
$40^{\circ}$ inci- \\
dence \\
Reflect- \\
ance, \\
Bureaud of \\
Stand- \\
ards in- \\
strument \\
$R_{\mathrm{U}}(40)$ \\
compare \\
with col- \\
umn 4
\end{tabular}} \\
\hline & & & & & & & & & & & \multirow{2}{*}{$\begin{array}{c}\text { Apparent } \\
\text { reflect- } \\
\text { ance } \\
A \mathrm{U}(0 ; 45)\end{array}$} & \multirow{2}{*}{$\begin{array}{l}\text { Reflect- } \\
\text { ance } \\
R_{\mathrm{V}}(0) \\
\text { compare } \\
\text { with col- } \\
\text { umn 3 }\end{array}$} & & \\
\hline & & $0^{\circ}$ & $35^{\circ}$ & $55^{\circ}$ & $60^{\circ}$ & & $0^{\circ}$ & $55^{\circ}$ & $60^{\circ}$ & & & & & \\
\hline 1 & 2 & 3 & 4 & 5 & 6 & 7 & 8 & 9 & 10 & 11 & 12 & 13 & 14 & 15 \\
\hline $\begin{array}{r}\text { Matt papers: } 1 \\
\text { M-1 } \\
\text { M-2 } \\
\text { M-5 } \\
\text { M-8 }\end{array}$ & $\begin{array}{r}0.870 \\
.517 \\
.201 \\
.045\end{array}$ & $\begin{array}{r}0.858 \\
.494 \\
.170 \\
.038\end{array}$ & $\begin{array}{r}0.864 \\
.501 \\
.180 \\
.040\end{array}$ & $\begin{array}{l}0.871 \\
.517 \\
.203 \\
.046\end{array}$ & $\begin{array}{r}0.872 \\
.525 \\
.213 \\
.048\end{array}$ & $\begin{array}{r}0.862 \\
.493 \\
.174 \\
.040\end{array}$ & $\begin{array}{r}\text { Per cent } \\
-1 \\
-4 \\
-15 \\
-16\end{array}$ & \begin{tabular}{r|} 
Per cent \\
0 \\
0 \\
+1 \\
+2
\end{tabular} & $\begin{array}{r}\text { Per cent } \\
0 \\
+1 \\
+6 \\
+7\end{array}$ & $\begin{array}{r}0.869 \\
.486 \\
.163 \\
.037\end{array}$ & $\begin{array}{r}0.872 \\
.496 \\
.165 \\
.037\end{array}$ & $\begin{array}{r}0.860 \\
.492 \\
.170 \\
.039\end{array}$ & $\begin{array}{r}0.498 \\
.184 \\
\hdashline\end{array}$ & $\begin{array}{r}0.865 \\
.511 \\
.187 \\
.055\end{array}$ \\
\hline $\begin{array}{l}\text { Glossy papers: }{ }^{2-} \\
\text { V-1 }\end{array}$ & & & & & & & & & & & & & & \\
\hline $\begin{array}{l}\mathrm{V}-1 \\
\mathrm{~V}-2 \\
\mathrm{~V}-\mathrm{-}\end{array}$ & $\begin{array}{l}.753 \\
.453 \\
283\end{array}$ & $\begin{array}{r}.735 \\
.440\end{array}$ & $\begin{array}{r}.740 \\
.431\end{array}$ & $\begin{array}{l}.745 \\
.434\end{array}$ &. .443 & $\begin{array}{r}.756 \\
.437\end{array}$ & $\begin{array}{l}-2 \\
-2\end{array}$ & $\begin{array}{l}-1 \\
-4 \\
-7\end{array}$ & $\begin{array}{r}0 \\
-2 \\
-3\end{array}$ & .412 & .707 & .736 & $\begin{array}{r}.730 \\
.438\end{array}$ & .742 \\
\hline $\begin{array}{l}V-5 \\
V-5\end{array}$ & .283 & .065 & $\begin{array}{l}.245 \\
.066\end{array}$ & .093 & $\begin{array}{l}.275 \\
.112\end{array}$ & .240 & $\begin{array}{l}-9 \\
-44\end{array}$ & $\begin{array}{c}-7 \\
-19\end{array}$ & $\begin{array}{l}-3 \\
-3\end{array}$ & $\begin{array}{l}.2019 \\
.019\end{array}$ & $\begin{array}{l}.214 \\
.019\end{array}$ & $\begin{array}{l}.255 \\
.067\end{array}$ & $\begin{array}{l}.254 \\
.071\end{array}$ & .246 \\
\hline $\begin{array}{l}\text { Magnesium carbonate }{ }^{3} \\
\text { Polain }\end{array}$ & .981 & .981 & .981 & .981 & .981 & .983 & $\begin{array}{r}0 \\
0\end{array}$ & $\begin{array}{r}0 \\
0 \\
0\end{array}$ & $\begin{array}{r}0 \\
0\end{array}$ & .995 & 1. 000 & .979 & .977 & .975 \\
\hline $\begin{array}{l}\text { Porcelain } \\
\text { White felt_... }\end{array}$ & .8000 & .786 & .793 & $\begin{array}{r}.803 \\
724\end{array}$ & $\begin{array}{r}.807 \\
734\end{array}$ & .784 & $\begin{array}{l}-2 \\
-8\end{array}$ & $\begin{array}{l}0 \\
0\end{array}$ & $\begin{array}{c}+1 \\
+1\end{array}$ & .791 & $\begin{array}{r}.793 \\
.692\end{array}$ & $\begin{array}{r}.785 \\
.674\end{array}$ & .793 & .798 \\
\hline Black felt 4 & .024 & .017 & .019 & .023 & .026 & .019 & -29 & -4 & +8 & .016 & .016 & .018 & .026 & .033 \\
\hline $\begin{array}{l}\text { Aluminum (ground surface) } \\
\text { Black glass: }\end{array}$ & .321 & .303 & .308 & .317 & .322 & .304 & -6 & -1 & 0 & .294 & .203 & .306 & & .297 \\
\hline $\begin{array}{l}\text { Ground surface- } \\
\text { Polished surface--............. } \\
\text { Silvered-glass mirror } 6\end{array}$ & $\begin{array}{l}.081 \\
.098 \\
.830\end{array}$ & $\begin{array}{l}.053 \\
.043 \\
.830\end{array}$ & $\begin{array}{l}.061 \\
.047 \\
.830\end{array}$ & $\begin{array}{l}.081 \\
.075 \\
.830\end{array}$ & $\begin{array}{l}.089 \\
.095 \\
.830\end{array}$ & $\begin{array}{l}.056 \\
.043 \\
.810\end{array}$ & $\begin{array}{l}-47 \\
-56 \\
0\end{array}$ & $\begin{array}{r}0 \\
-23 \\
0\end{array}$ & $\begin{array}{r}+10 \\
-3 \\
0\end{array}$ & .0465 & .047 & .054 & .077 & $\begin{array}{l}.063 \\
.066\end{array}$ \\
\hline Deted-gras & & & & & & & & & & & & & & \\
\hline
\end{tabular}




\section{DISCUSSION OF DATA}

The apparent reflectances for the diffused illumination represented by the brightness Distribution III ${ }^{20}$ (fig. 4), are shown by the circles and curves in Figure 5. Measurements on some of the samples were also made for brightness Distributions I and II of Figure 4. These measurements were found, however, to be slightly incorrect in absolute value, due to stray light errors ${ }^{21}$ in the brightness measurements. The data were subsequently corrected to give the same reflectance as obtained with Distribution III, for which, it is believed, no stray light errors were present. The corrected data are shown by the triangles and crosses, respectively, in Figure 5. As may be noted, there is good agreement between the relative values of the apparent reflectances for any one sample for each of the three different brightness distributions over the source, showing that the diffused illumination on the sample, resulting from each of these brightness distributions, is a sufficient approximation to the ideal completely diffused type of illumination for practical purposes. The three different angular distributions of incident luminous flux give sensibly the same distribution of the reflected flux and undoubtedly the same absolute value of the reflectance.

A comparison of columns 3 and 13 (Table 2) shows the equality of the unidirectional normal reflectance and the apparent diffuse reflectance for normal observation, in accordance with the reciprocal relation expressed in equation (15). Assuming this relation, then, the ordinates of the curves of Figure 5 give (as previously stated) the unidirectional reflectances for all angles of incidence. The reflectance for any type of multidirectional illumination must be given by taking a (properly weighted) mean value of the unidirectional reflectances; hence these data as a whole give some information as to the range of variation which may occur between the reflectances for various types of illumination with different kinds of materials. Thus, the reflectances for the black glass mirror or for the sample $\mathrm{V}-5$ will vary under different types of illumination from a small fraction up to unity. In the case of magnesium carbonate the reflectance is (practically) independent of the manner of illumination. Its reflectance is so high, however, as to leave very little range for an appreciable variation. It would be of interest to know if a more

\footnotetext{
${ }^{20}$ In case of the mirror samples and the hemispherical source, the actual brightness distribution along the zero meridian was used to obtain the apparent reflectances.

21 These measurements were made without the tubes $T$ and the diaphragm $D^{\prime}$ (fig. 3). Consequently a considerable amount of stray light was present in the photometric field, necessitating a stray light correction to all the brightness measurements. This correction could not be determined with a high degree of accuracy. Assuming an error was made in the evaluation of the stray light, the reflectances determined by either Distribution I or II should bear a linear relation to the reflectances determined for Distribution III (for which all stray light effects were practically eliminated by insertion of the tubes $T^{\prime}$ and diaphragm $D^{\prime}$ ). This was found to be the case, and it may be presumed that, had the stray light error been exactly evaluated, the same absolute values of reflectance would have been obtained for Distributions I and II as were obtained or Distribution III.
} 
highly absorbing medium could be constructed for which the reflectance would be practically independent of the manner of incidence. If, in addition, the reflectance was independent of the spectral composition of the incident light an ideal and much needed material would be available for reflection standards.

Vertical arrows on the curves of Figures 5 and 6 mark the mean ordinates (by equations (19) and (21)) and show the angles of observation for which the apparent reflectance is equal to the reflectance. In the case of completely diffused illumination, these angles are in the range between 50 and $55^{\circ}$ for matt samples, increasing to $60^{\circ}$ or above for glossy samples.

Similar data for unidrectional normal illumination has been given by Taylor, ${ }^{22}$ who measured the brightness of 25 or 30 different samples at various angles of view, and found that the apparent reflectance for an angle of observation of $50^{\circ}$ was equal to the reflectance to within 1 per cent for most of his samples. An angle of about $59^{\circ}$ was found, however, for polished milk glass.

A very useful purpose would be served in reflectometry if this angle was more nearly the same for all kinds of samples, for then a single brightness measurement at the proper angle (for either type of symmetrical illumination) might yield the true reflectance to a sufficient approximation. In the case of unidirectional normal illumination and samples of fairly high reflectance (magnesium carbonate, porcelain, white felt, $\mathrm{M}-1$ and $\mathrm{M}-2$ ), it may be noted (fig. 6) that the apparent reflectance for an observation angle of $50^{\circ}$ is within 1 per cent of the reflectance; but it is evident that a single brightness measurement at any one specified angle can not be generally relied upon as a sufficiently approximate method of reflectometry. The degree of approximation which may be obtained in the case of diffused illumination is shown in columns 8, 9, and 10 of Table 2 and considered later.

Samples V-1, V-2, V-3, and V-5 have a very smooth surface and consequently a distinct specular component of reflectance (fig. 7) superposed on the diffuse component. This specular component amounts to approximately 4.7 per cent of the incident light for nearly normal incidence. Its effect on the shifting of the mean ordinate ${ }^{23}$ for unidirectional normal illumination is shown in Figure 6 , whereas the mean ordinate for completely diffused illumination remains close to $60^{\circ}$ for these samples. The magnitude of the

\footnotetext{
${ }_{22}$ Trans., III. Eng. Soc., $15^{2}$, p. $813 ; 1920$. The quantity given by Taylor is the ratio of the apparent reflectance to the reflectance, and expressed in his paper (fig. 4) as the "ratio of specific brightness of test surface to the brightness of a perfect diffusor radiating the same total flux."

${ }^{23}$ In the calculation of the refiectance of these samples for unidirectional normal illumination, the diffuse component was obtained separately using equation (22) and ignoring the component of the apparent reflectance for $\theta^{\prime}=0$ which is due to the specularly reflected light. The specular component of the reflectance was measured separately, using a small diffusely emitting surface as a source which was just large enough to completely fill the photometer field with light. The brightness of this source was first observed directly and then as seen by specular reflection from the surface of the sample.
} 
specular component is governed by the Fresnel reflection formulas. Hence, under unidirectional illumination, this component increases with the angle of incidence (while the diffuse component decreases), approaching the value of unity at grazing incidence, as the type of reflection becomes entirely specular. For completely diffused illumination the apparent reflectance of these samples consequently approaches unity for large angles of observation, but approaches a value less than unity for the other samples (having a rough surface).

It may be noted that the apparent reflectances of the mirror samples and the $\mathrm{V}$ samples do not appear in Figures 6 and 7 for angles of observation corresponding to the angle of specular reflection. A consideration of the definitions of brightness, apparent reflectance, and unidirectional illumination previously given, together with the well-known laws of reflection from a mirror surface, shows that the apparent reflectance for unidirectional illumination and purely specular reflection (as from a perfect mirror surface) has a value at one angle only and that value is indefinitely large. In the case of the $\mathrm{V}$ samples, as illustrated in Figure 7 for $45^{\circ}$ illumination, the diffuse component of the apparent reflectance (roughly considered as due to light reflected from the body of the material only) for $\theta^{\prime}=-45^{\circ}$ has a finite value, which can not be separated in the observations, however, from the value of the specular component (regarded as due to surface reflection only). The broadness of the peaks on these curves (at $\theta^{\prime}=-45^{\circ}$ ) is due mainly to the finite and fixed solid angular extent of the incident luminous flux, but may also be due in part to the finite solid angular extent of the beam taken off the sample by the photometer. The illumination is no longer unidirectional (in strict accordance with the definition) for the case of specular reflection. If the surface of these samples was perfectly smooth and the solid angular extent of the incident flux allowed to decrease indefinitely (to satisfy the definition for unidirectional illumination), the bands (partly) shown in Figure 7 would become narrower and higher (presuming a satisfactory measuring instrument is used) and the value of the specular component of the apparent reflectance indefinitely large. Since no surface in nature is a perfect mirror, the apparent reflectance will always be finite, but may be difficult to measure in the cases considered. For angles of observation close to $\theta^{\prime}=-45^{\circ}$, the brightness results from a mixture of both body and surface reflection. If the specular reflection could be entirely ignored in the measurements, the curve for the diffuse component would probably take a form through this region as indicated by the dotted line in the case of the V-2 sample of Figure 7.

The slope of the curves for completely diffused illumination is opposite to that predicted by Walsh (loc. cit.) for his theoretical 
samples. His simple empirical formula, however, only roughly represents the reflective properties of materials. Furthermore, it violates the general reciprocity condition previously stated in equation (14). Consequently, the results derived by integration of the formula do not satisfy the integral reciprocal relation expressed in equation (15). Thus, the formula makes the unidirectional reflectances independent of the direction of incidence while it allows the apparant diffuse reflectances to vary with the direction of observation, and the calculation of the brightness variation with angle of observation (for completely diffused illumination) shows a continuously decreasing brightness with increasing angle. This result is used by Walsh to explain the low value (0.88) obtained by Nutting ${ }^{24}$ for the reflectance of magnesium carbonate. His contention that this low value is due to a decrease in brightness of the carbonate for large angles of observation is untenable in view of the present data, for the brightness of magnesium carbonate at large angles of observation $\left(75^{\circ}\right)$ is sensibly the same as at smaller angles. The Nutting reflectometer theoretically measures the apparent reflectance for completely diffused illumination for an angle of observation of approximately $75^{\circ}$. The results obtained with this instrument would, in general, be higher than the true reflectance, but it should give the correct value for magnesium carbonate. The most probable explanation of the low value actually obtained for this substance lies in the effect of the departure of the illumination on the sample from the completely diffused type and, more particularly, its incorrect evaluation by a single brightness measurement of the source.

The curves in Figures 5, 6, and 7 are of further interest in methods of colorimetry, since they afford a comparison of the relative brightnesses of the samples under different methods of illumination. In columns 3, 11, and 12 of Table 2 the apparent reflectances (proportional to brightnesses) are tabulated for the following cases: (1) Completely diffused illumination, normal observation; (2) unidirectional normal illumination, $45^{\circ}$ observation; (3) unidirectional $45^{\circ}$ illumination, normal observation. The values for cases (2) and (3) are practically identical, ${ }^{25}$ as they should be in accordance with the reciprocity law stated in equation (14).

\footnotetext{
${ }^{24}$ Nutting, Trans., IIl. Eng. Soc., \%, p. 412; 1912. See also Karrer, B. S. Sci. Paper No. 415, p. 210; August. 1921. The principle of Nutting's method is that of two parallel infinite planes, one of which is the source and is assumed to obey the cosine law of emission. The other plane is the sample, the reflectance of which is to be measured. If the sample is a perfect diffusor, the relative brightnesses of the two planes gives the reflectance for completely difiused illumination.

${ }^{25} \mathrm{By}$ the general reciproeity law (footnote 11), these two methods of illumination and observation should also be equivalent as regards spectral composition and polarization of the reflected light.
} 
By the same relation the curves of Figure 6 can be interpreted as the apparent reflectance of the samples for normal observation and various directions of unidirectional illumination. Similarly, the

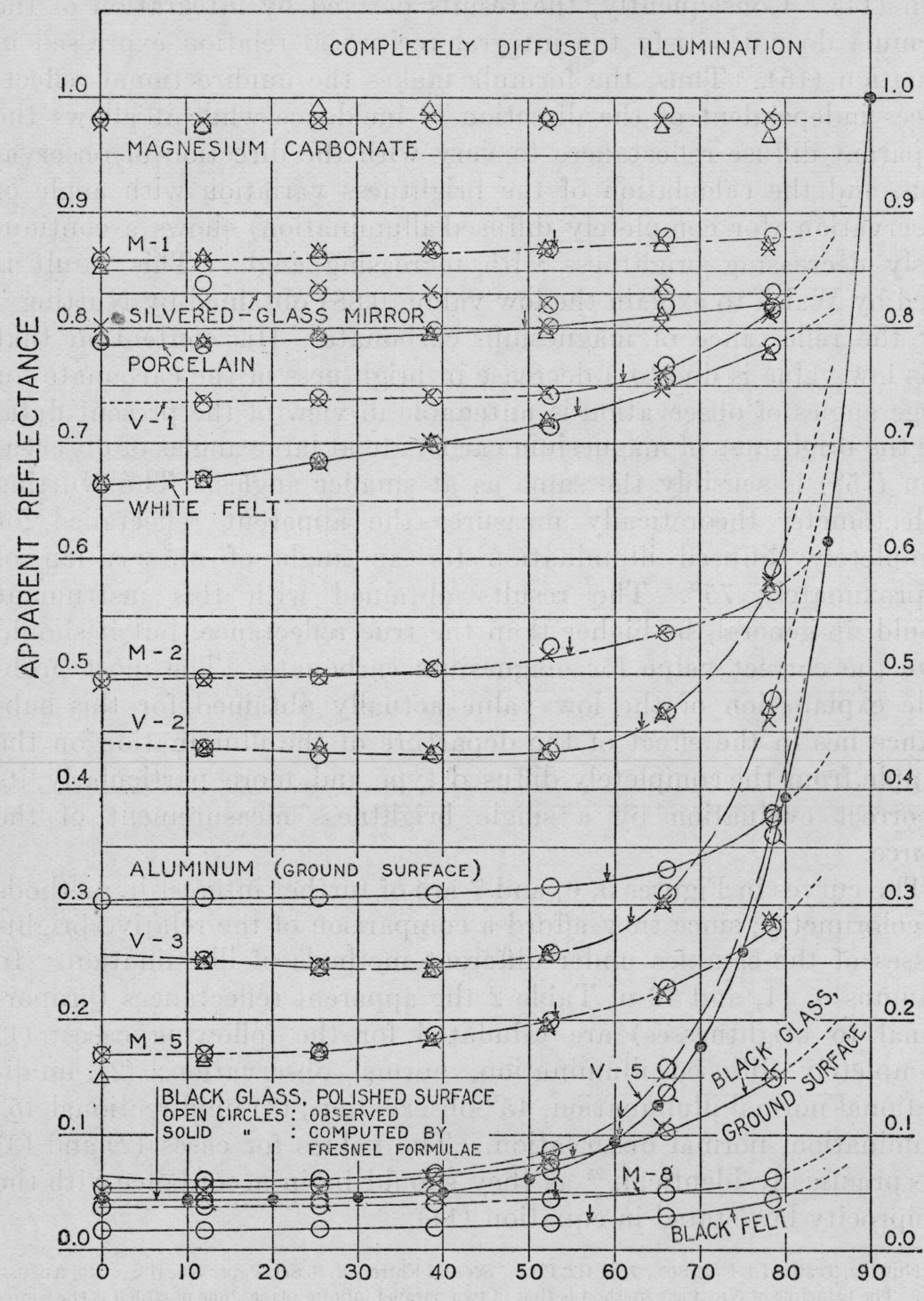

ANGLE FROM NORMAL, $\theta^{\prime}$.

FIG. 5.-Apparent reflectances for completely diffused illumination

Vertical arrows on curves mark angles of observation for which the apparent reflectance is equal to the reflectance. (Reciprocal interpretation of curves: Reflectances for unidirectional illumination.)

curves of Figure 7 give the apparent reflectances for $45^{\circ}$ observation and unidirectional illumination at various angles of incidence in the plane of observation. 


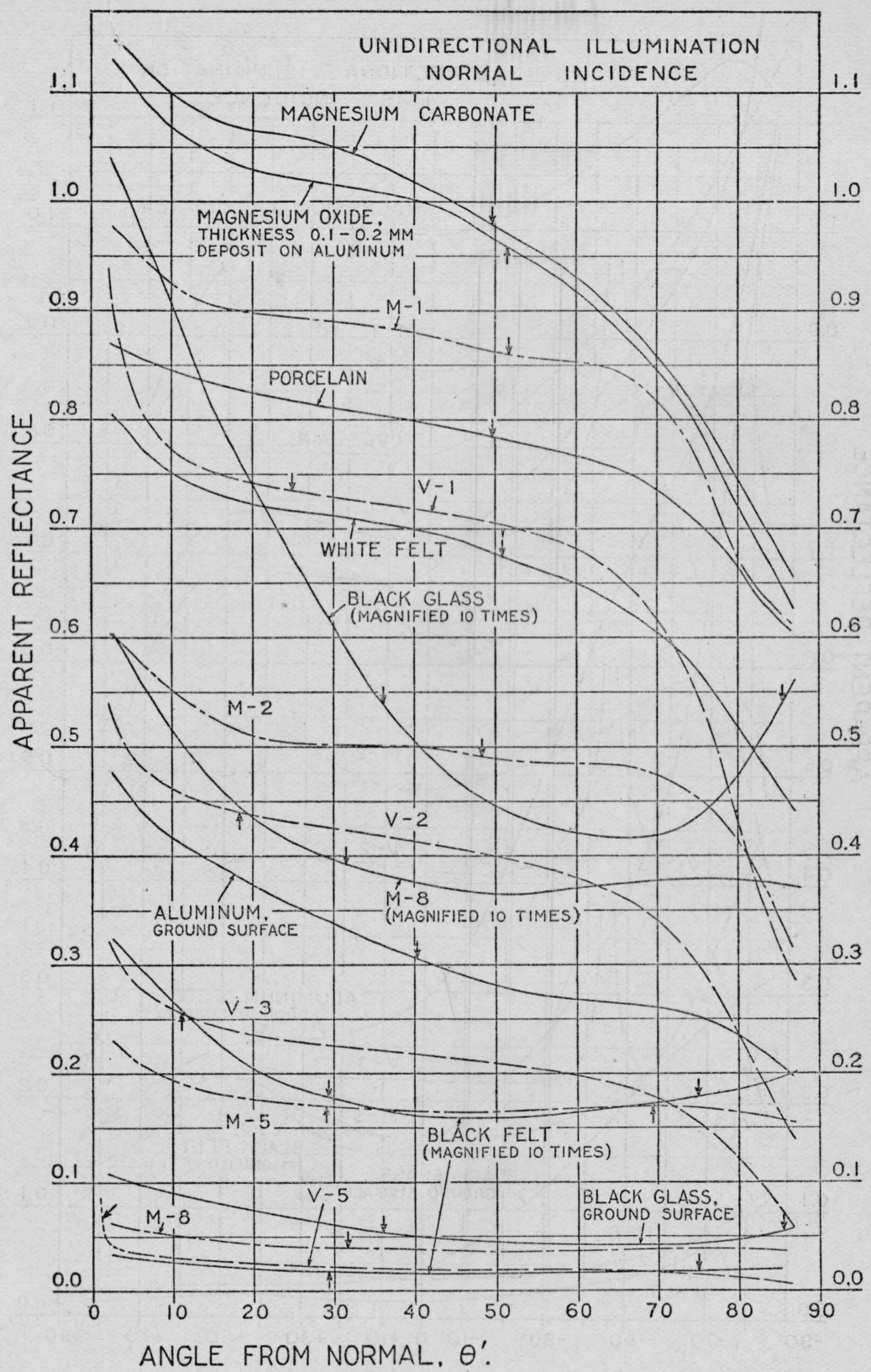

FIG. 6.-Apparent reflectances for unidirectional normal illumination

Vertical arrows on curves mark angles of observation for which the apparent reflectance is equal to the reflectance. (Reciprocal interpretation of curves: Apparent reflectances for normal observation and various angles of unidirectional illumination.) 


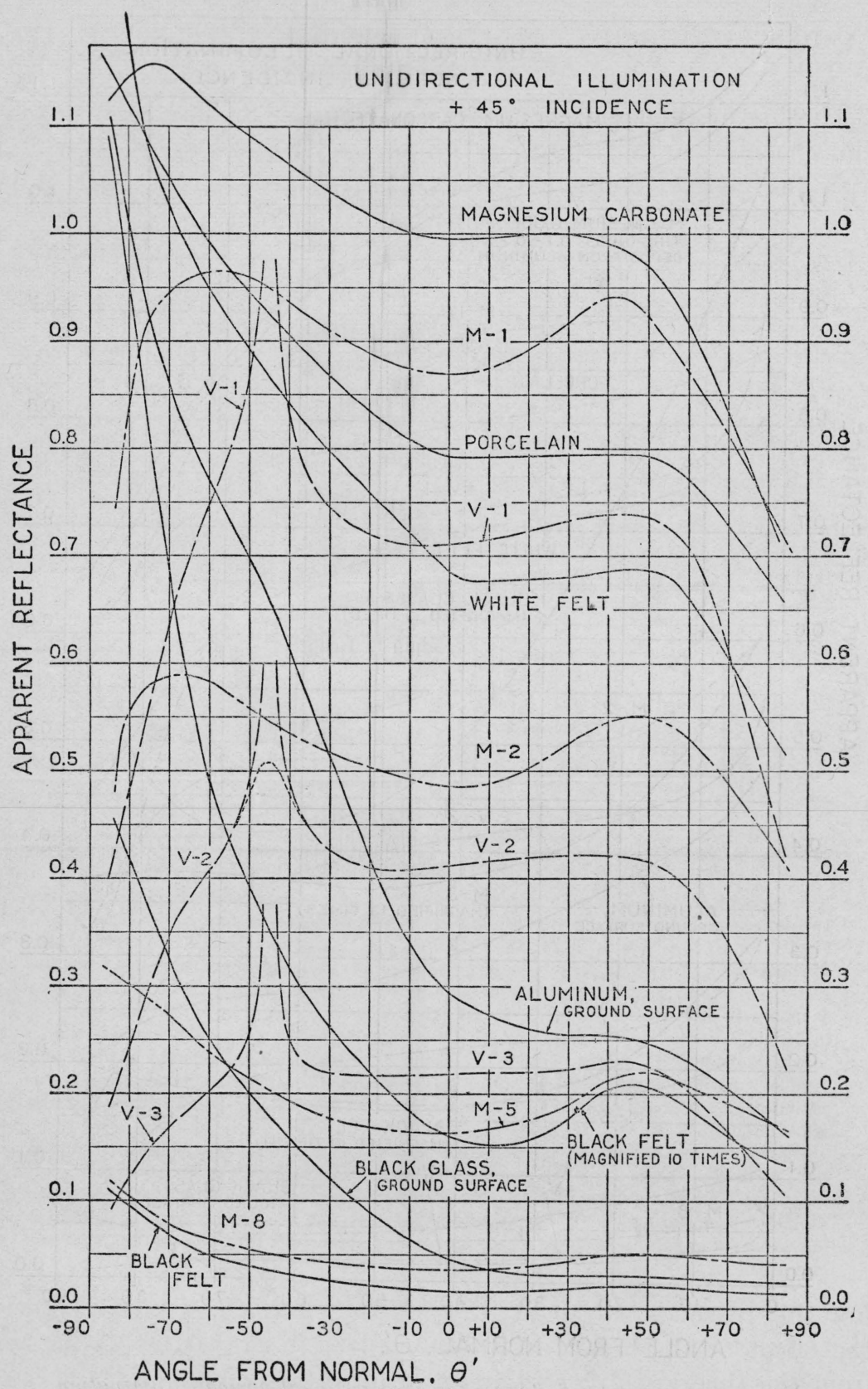

FIG. 7.-Apparent reflectances for unidirectional $45^{\circ}$ illumination, and angles of observation in the plane of incidence

(Reciprocal interpretation: Apparent reflectances for $45^{\circ}$ observation and unidirectional illumination at angles in the plane of observation.) 


\section{USE OF SPHERE REFLECTOMETER}

\section{DESCRIPTION OF DIFFUSED-ILLUMINATION REFLECTOMETER AND MEASUREMENTS}

The use of the sphere as a device to obtain a completely diffused illumination on the sample is of particular interest in connection with the general method of reflectometry described in Section IV.

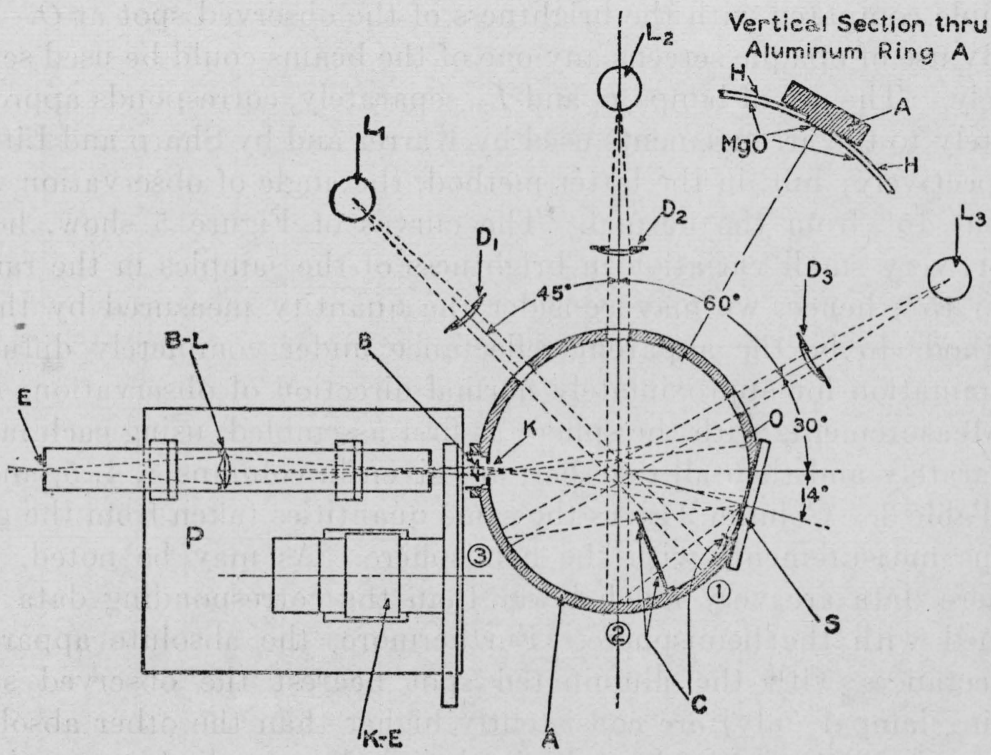

FiG. 8.-Diagram of sphere reflectometer

$A$, horizontal section through center of aluminum ring, $20 \mathrm{~cm}$ inside diameter, which supports two iron hemispheres. $S$, position of sample; opening $2 \mathrm{~cm}$ square into sphere. $L_{1}, L_{2}, L_{3}$, 9 volt 27 c. p. Mazda lamps. $D_{1}, D_{2}, D_{3}$, lenses, each 10 diopters, placed $12 \mathrm{~cm}$ from lamp filaments. $1,2,3$, position of illuminated spots on sphere wall; size approximately $15 \mathrm{~mm}$ diameter. $C$, screens to shade sample from illuminated spots. $P$, photometric apparatus; same as used with hemisphere. See also Figure 2. $O$, position of observed spot. $K$, aperture $6 \mathrm{~mm}$ in diameter. Other apertures into sphere, through which beams 1,2 , and 3 enter, are each 15 $\mathrm{mm}$ in diameter. $B$, biprism used to obtain greater separation between observed spot and sample than would be obtained with biprism at $B-L$ alone. These prisms were taken from material on hand. A single biprism at $B-L$ of the proper refracting angle would accomplish the same purpose.

The center of the illuminated and observed spots, and all apertures into the sphere, lie in the same horizontal plane. To obtain a small intensely illuminated spot with small aperture into the sphere, a projection system with condenser and objective lenses should be used. The above simple arrangement has served the purpose well enough, but the illumination on the sample (using one lamp only) was not sufficient for the best photometric precision in the brightness measurements.

For this reason a thorough study of the sphere was made, showing the effect of the departure of the reflective properties of the wall from the theoretical conditions previously assumed.

A sphere was built up of two iron hemispheres fitted into a metal ring $A$ (fig. 8). A horizontal section through center of the ring $A$, light sources, and sample is shown in the figure. The interior of the sphere was first coated with aluminum paint and then covered. 
with a deposit of magnesium oxide. Three beams of light were projected into the sphere, forming the directly illuminated spots at positions 1,2 , and 3 . These spot sources are shaded from the sample by screens $C$ and situated at angular distances of 60,38 , and $8^{\circ}$, respectively, from the normal to the observed spot at $O$. The same photometric apparatus $P$, previously used with the hemisphere (fig. $3)$, could be transferred to the sphere, and the brightness of the sample compared with the brightness of the observed spot at $O$.

By use of opaque screens any one of the beams could be used separately. The use of lamps $L_{1}$ and $L_{2}$, separately, corresponds approximately to the arrangements used by Karrer and by Sharp and Little, respectively; but, in the latter method, the angle of observation was about $15^{\circ}$ from the normal. The curves of Figure 5 show, however, very small variation in brightness of the samples in the range 0 to $15^{\circ}$; hence, we may consider the quantity measured by these methods to be the apparent reflectance under completely diffused illumination for approximately normal direction of observation.

Measurements with the sphere as first assembled, using each lamp separately and then all together, are given in columns $3,4,5$, and 6 of Table 3. Column 2 gives the same quantities taken from the previous measurements with the hemisphere. As may be noted, the sphere data are very much lower than the corresponding data obtained with the hemisphere. Furthermore, the absolute apparent reflectances with the illuminated spot nearest the observed spot (using lamp 1 only) are consistently higher than the other absolute values; and absolute values obtained with all lamps lie between those for lamps 1 and 2, respectively. The use of all lamps together tends to give a more uniform distribution of brightness over the sphere wall and to eliminate the effect of the position of the illuminated spot.

After these measurements were made it was noticed that the lower half of the sphere and central ring appeared just perceptibly brighter than the upper hemisphere. The lower hemisphere and central ring had been coated as a unit with magnesium oxide and had, apparently, received a heavier deposit than the upper hemisphere, so that a considerable difference probably existed between the reflectances of the two parts. The upper hemisphere was consequently removed and given an additional deposit of oxide. This operation did not alter the diffuse reflective properties of the illuminated or observed spot, since they are all located on the central ring. To the unaided eye, placed at the sample opening, the interior of the sphere now appeared to be of uniform brightness.

The result was a marked increase in the numbers found for the absolute apparent reflectances. Measurements on samples $\mathrm{M}-1, \mathrm{~V}-1$, porcelain, and magnesium carbonate, showed decreasing values (as 
in previous case) for lamps 1,2, and 3, used separately; but the extreme differences (between lamps 1 and 3 ) were reduced approximately 30 per cent. The average difference between values for lamps 1 and 3 was now only 3 per cent. The data for all samples, using all lamps together, are given in column 7 of Table 3. These values are very nearly equal (within 1 per cent) to the absolute values obtained with lamp 2 used alone (after recoating the upper hemisphere), but are 6 per cent lower than the corresponding data obtained with the hemisphere. These discrepancies are the result of incorrect conditions still existing in the sphere. The matter is fully discussed in the following section.

$2284^{\circ}-28-5$ 
TABLE 3.-Measurements of apparent reflectance, $A_{\mathrm{D}}(7)$, with sphere reflectometer

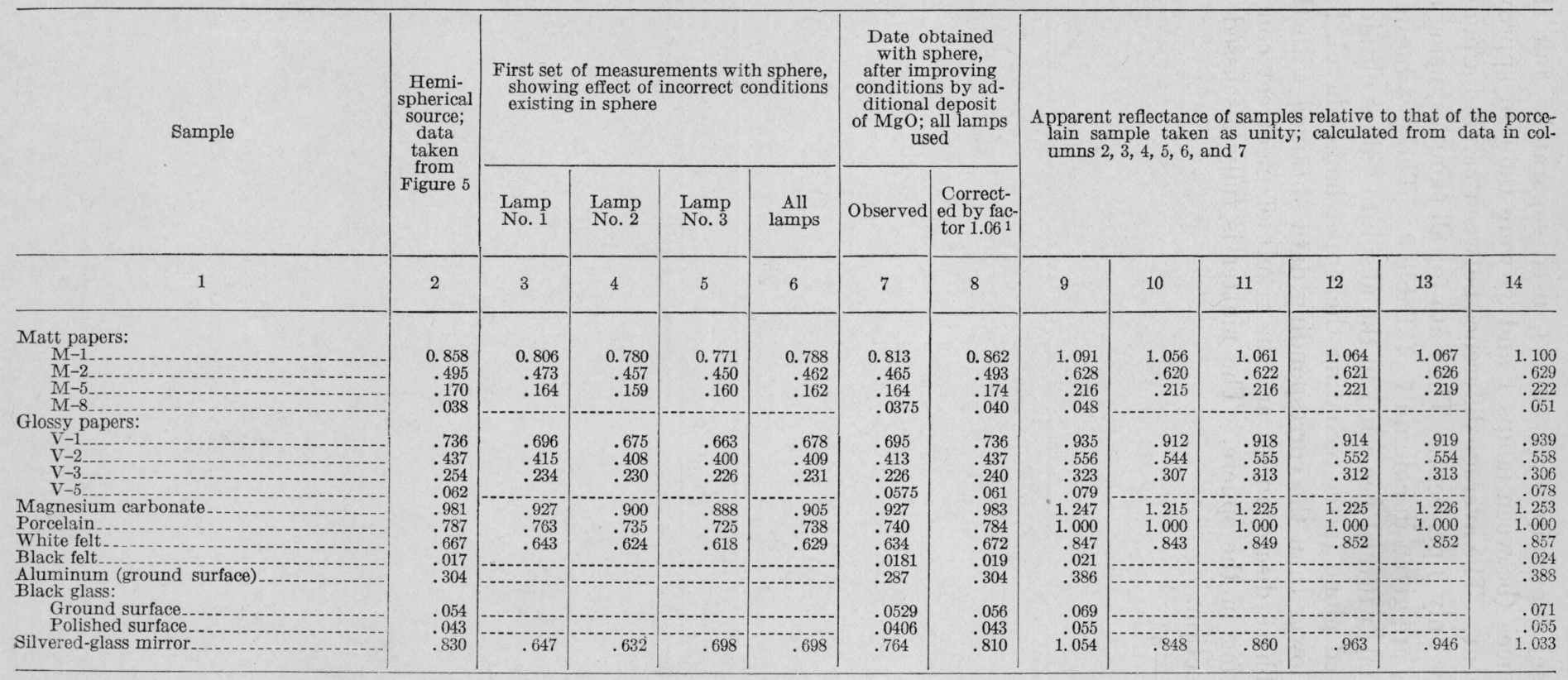
1 The factor 1.06 is the ratio of the brightness of the observed spot on the sphere wall to the "equivalent-hemisphere" brightness of the spherical source. It is a factor belonging
to the sphere and insuring a correct evaluation of the total illumination on the sample by means of a single observation of the brightness of the sphere wall. (See full explanation to the sph
in text.) 


\section{EVALUATION OF ILLUMINATION ON SAMPLE}

The differences between the apparent reflectances obtained with the sphere and hemisphere may be explained by the departure of the sphere wall from the theoretical conditions previously assumed. The unfulfilled conditions are (1) the departure of the sphere wall from a perfect diffusor, and (2) the variation of the reflectance over different parts of the sphere.

They are not independent in their effect on the measurements. The first defect causes variations in the brightness of the observed spot (and other parts of the sphere wall) corresponding to different positions of the illuminated spot. The magnitude of these variations depends also on the second defect but decreases as the average reflectance of the wall increases. If the sphere wall is a perfect diffusor but of varying reflectance, the position of the illuminated spot has no effect whatever on the brightness distribution over the sphere or on the measurements of apparent reflectance. The second defect causes a constant error in the measurement of the apparent reflectance for all samples. This error varies with the first defect, but exists even when the sphere wall is a perfect diffusor. The relative magnitude of these effects and the possibility of their elimination will now be considered in connection with the experimental data.

As a consequence of the departure of the sphere wall from a perfect diffusor, the brightness of the observed spot (hence the apparent reflectance of a sample) varies with the position of the illuminated spot. The relative brightnesses of the observed spot, when lamps $L_{1}, L_{2}$, and $L_{3}$ are used separately, can be determined approximately from the construction of the sphere reflectometer (fig. 8), the geometry and properties of the sphere itself, and the reflective properties of the magnesium oxide surface.

Measurements on films of magnesium oxide ${ }^{26}$ deposited on aluminum have been made with the equipment herein described. Data for unidirectional normal illumination are given in Figure 6 . In the following discussion the ordinates of this curve are taken to represent the relative brightnesses of an illuminated spot on the sphere walleven though the thicknesses and reflectances of the oxide layer may be different in the two cases. Brightness measurements for completely diffused illumination were made on a film approximately 0.3 $\mathrm{mm}$ thick deposited on aluminum. The brightness was found to remain constant for various angles of view (as in the case of magnesium carbonate), and the measurements show that a sufficiently thick layer

${ }^{20}$ In the selection of samples for this investigation only materials likely to remain permanent in their reflective properties were chosen. It is known that the reflectance of a magnesium oxide film varies with the thickness of deposit and, for thin layers, with the reflectance of the material upon which it is deposited. The film is also very fragile and of questionable permanence in reflective properties. For these reasons, and because a magnesium carbonate block served equally well as a good diffusor of high reflectance, a magnesium oxide sample was not included in the regular series of measurements (which have extended over a period of several months). 
of the oxide has a very high reflectance, practically the same as that found for the carbonate.

The following steps may be noted in the determination of the effect of the position of the illuminated spot:

1. The brightness of the observed spot is always compared in the photometer with the brightness of another part of the sphere wall (occupied by a sample). Consequently, the relative brightnesses (photometer readings) of the observed spot, when lamps $L_{1}, L_{2}$, and $L_{3}$ are used separately, do not depend on the constancy or total quantity of the light projected into the sphere from each lamp. Furthermore, the light from each lamp is incident normally on the sphere wall and is diffusely reflected in the same manner from each illuminated spot. Hence, for the purpose in view, the spot sources 1,2 , and 3 may be regarded as equal in all respects.

2. The total illumination $E$ of the observed spot, due to either of the spot sources 1,2 , or 3 , used separately, may be divided into three components:

$$
E=E_{\mathrm{a}}+E_{\mathrm{b}}+E_{\mathrm{c}}
$$

where $E_{\mathrm{a}}$ is received directly from the illuminated spot, $E_{\mathrm{b}}$ is contributed by the first reflection of the direct illumination of all other parts of the sphere, and $E_{\mathrm{c}}$ results from the multiple reflections of $E_{\mathrm{b}}$.

3. The difference in brightness of the observed spot, when viewed normal to its surface and nearly normal (as in fig. 8), is assumed to be negligible for each of the three components of illumination.

4. Lines connecting the spot sources 1,2 , and 3 with the observed spot at $O$ (fig. 8 ) make angles of 60,38 , and $8^{\circ}$, respectively, with the normal at $O$, and also make the same angles with the corresponding normals to the spot sources.

5. From the geometry of the sphere the illumination of the observed spot, due to the component $E_{\mathrm{a}}$ from either of the spot sources 1,2 , or 3 is independent of the distance of this source from the observed spot.

6. Owing to the departure of the magnesium oxide surface from a perfect diffusor (for unidirectional normal illumination), the direct illumination of the observed spot is different, however, for sources 1 , 2 , or 3 , and proportional to the ordinates of the curve (in fig. 6) for angles 60,38 , and $8^{\circ}$, respectively. Recalling the reciprocal interpretation of the curves in Figure 6 , it is evident that the normal brightnesses of the observed spot, due to spot sources 1,2 , and 3 , used separately, are in the proportion $(0.916)^{2}:(1.005)^{2}:(1.083)^{2}$, respectively.

If the magnesium oxide surface were a perfect diffusor with the same reflectance for unidirectional normal illumination, these brightnesses would be the same in all three cases and, on the same brightness scale, would have the value $(0.957)^{2}$, where 0.957 is the mean ordinate of the curve for magnesium oxide. 
7. It has been shown by others ${ }^{27}$ (assuming perfect diffusion in the sphere) that

$$
\frac{E_{\mathrm{a}}}{E}=1-R
$$

where $R$ is the average reflectance of the sphere wall. By inspection of the interior surface of the sphere wall and comparison with the deposit of magnesium oxide measured (fig. 6), an estimate of 0.92 for the average reflectance of the sphere wall seemed reasonable. Then the component $E_{\mathrm{a}}$ is 8 per cent of the total illumination.

8. The magnitude of the second component is given by

$$
\frac{E_{\mathrm{b}}}{E-E_{\mathrm{a}}}=1-R
$$

or

$$
\frac{E_{\mathrm{b}}}{E}=(1-R) R=0.074
$$

The second component is shown to be small and, while not completely diffused, is incident on both sample and observed spot. Compared with the first component its effect on the relative brightnesses is certainly negligible. Components $E_{\mathrm{b}}$ and $E_{\mathrm{c}}$ together represent 92 per cent of the total illumination of the observed spot and may be regarded here as completely diffused.

9. The experimental data on magnesium oxide for completely diffused illumination show that its brightness does not vary appreciably with the angle of view. Consequently, the sphere wall acts as a perfect diffusor for the components $E_{\mathrm{b}}$ and $E_{\mathrm{c}}$, and the reflectance is the same for both unidirectional and diffused illumination.

Adding 8 per cent of the brightness due to component $E_{\mathrm{a}}$ alone to 92 per cent of the brightness $E_{\mathrm{a}}$ would give if the sphere wall acted as a perfect diffusor for this component (as it does for components $E_{\mathrm{b}}$ and $E_{\mathrm{c}}$ ), the relative brightnesses of the observed spot, due to the total illumination received from spot sources 1,2 , and 3 , used separately, are found to be in the proportion $(0.910):(0.924):(0.937)$.

The above determination is not presumed to be accurate. It is a probable explanation, however, of some of the experimental results, for it is evident from the definition of the apparent reflectance that these calculated differences in the brightness of the observed spot will account for the observed difference of 3 per cent between the absolute apparent reflectances measured with lamps 1,2, and 3, used separately (after the additional deposit of magnesium oxide had been made). The calculations and observations show that the position of the illuminated spot relative to that of the observed spot may have an appreciable effect on the measurements with the sphere when the

\footnotetext{
${ }^{27}$ See footnote 16.
} 
average reflectance of the sphere wall is not very high. Although it is impossible to make the sphere wall a perfect diffusor for all types of illumination, it is possible to make the average reflectance high enough to render the effect negligible. The additional deposits of magnesium oxide, referred to in the description of the experiments with the sphere, made the reflectance of the sphere wall more uniform over all its parts and increased its average value. The component $E_{\mathrm{a}}$ was unaltered, $E_{\mathrm{b}}$ was increased slightly, but $E_{\mathrm{c}}$ was increased very much more, thus decreasing the effect of the relative positions of the illuminated and observed spots.

Consider, now, the effect due primarily to the nonuniform reflectance over the sphere wall. The brightness distribution over the sphere depends directly on the reflectance of the various parts as well as on the diffusing properties of the wall. In the use of the sphere the brightness of the observed spot is taken as a measure of the illumination on the sample. Obviously, then, this brightness (observed from the position of the photometer) must be equal to the equivalent-hemisphere brightness of the spherical source (as viewed from the sample), otherwise the measurements will be in error by a constant factor for all samples. The approximately constant difference observed between the sphere data and the hemisphere data (Table 3, columns 7 and 2) suggests that this incorrect condition may exist in the sphere reflectometer herein described. The ratio of the equivalent-hemisphere brightness of the sphere to the brightness of the observed spot was directly determined (for the case of all lamps) by the same procedure followed previously with the hemispherical source.

Using a mirror of known reflectance, the brightness at various points on the sphere wall relative to the brightness of the observed spot was measured with the photometer, sets of measurements being made at angles of approximately 10,20,30, 40, and $60^{\circ}$, respectively, from the normal to the plane of the sample holder. It may be noted that in this way only little more than half of the sphere wall opposite the sample was covered. However, the region in the neighborhood of $45^{\circ}$ is most effective in determining the illumination on the sample. This fact is indicated by the large value which the factor $K(\theta)$ (see equations (17) and (18)) takes for angles in this region. In the case of the hemisphere these results are the combined (opposing) effects of two physical conditionsfirst, the increase in area of zones as $\theta$ increases, and, second, the decrease in component of illumination from each zone which results from the approach to grazing incidence. In the case of the sphere there is the further complication of variation in distance from the several elements of the sphere wall, but the general effect is the same in that the zones in the neighborhood of $45^{\circ}$ remain most effective. 
Using equation (17), a value of 0.94 was obtained from these measurements, showing that the brightness of the observed spot ${ }^{28}$ was 6 per cent higher than the equivalent-hemisphere brightness of the whole sphere. The directly measured brightness ratios in Table 3 , column 7 , were then corrected by a factor 1.06 , which thus completely eliminates the error in the evaluation of the total illumination on the sample resulting from a nonuniform brightness distribution over the sphere wall. The corrected values are tabulated in Table 3 , column 8 , and Table 2 , column 7 , and are in good agreement with the corresponding data obtained by the other methods (compare with Table 2, columns 3 and 11).

It is of interest to compare the relative apparent reflectances of the samples, as expressed in terms of the apparent reflectance of the porcelain sample (chosen arbitrarily), and measured under the different approximations to completely diffused illumination and normal observation obtained with the sphere and hemisphere. These data are given in the last six columns of Table 3. The general agreement of values for all samples (excepting the mirror samples) shows that the difference between the brightness of the observed spot and the equivalent-hemisphere brightness of the entire sphere wall is a greater source of error than any change in the normal brightness of the sample resulting from the corresponding variation in the angular distribution of the incident light from the ideal completely diffused type of illumination. Hence, for relative apparent reflectance measurements, the illumination in all these cases seems to be a sufficient approximation to complete diffusion for practical purposes. To make absolute measurements, however, one must be assured that the brightness of the observed spot is a correct measure of the total illumination on the sample.

If the variations from the ideal conditions desired in the sphere are not too large, then the total effect (again excepting the mirror samples) may be expressed by the ratio of the brightness of the observed spot to the equivalent-hemisphere brightness of the sphere. This ratio is a correction factor belonging to the sphere reflectometer and insuring a correct evaluation of the illumination on the sample by means of a single observation of the brightness of the sphere wall.

\section{ERRORS IN MEASUREMENT OF REFLECTED LIGHT}

In the case of the mirror samples and samples having a fairly distinct specular component of reflectance with little or no diffuse component, the correction factor above determined may cease to

\footnotetext{
${ }^{28}$ An examination of the interior of the sphere after the measurements were completed showed a thicker deposit of the oxide in the region of the observed spot than over the greater part of the remaining surface; hence, the reflectance may be appreciably higher in this region. The effect of the departure of the magnesium oxide from the perfect diffusor will also result in a slightly higher illumination on the central ring opposite the directly illuminated spots. These observations are in qualitative agreement with the results of the brightness distribution measurements.
} 
cover adequately the total effect of the departure of the sphere wall from the desired conditions. The illumination may not be a sufficient approximation to complete diffusion for these samples. Only a very limited region of the source contributes to the light reflected into the photometer, and if the brightness of this region is not equal to the equivalent-hemisphere brightness of the source another correction factor must be applied, which is the ratio of the equivalent-hemisphere brightness of the source to the brightness of that area of the source actually used. This second correction factor reduces the evaluation of the reflected light (as given by the photometer) to the value which would be obtained if the illumination on the sample were completely diffused. This correction was not applied to the data in Table 3.

The two correction factors defined may obviously be combined into a single factor (for mirror samples) which is the ratio of the brightness of the observed spot to the brightness of that part of the sphere source actually contributing to the light entering the photometer.

Assuming, again, that all theoretical conditions affecting the angular distribution of the incident luminous flux and its correct evaluation have been satisfactorily realized, the variation in brightness of the sample for different angles of view remains as a source of error in the evaluation of the total reflected flux - by means of a single brightness measurement of the sample.

An approximation to the true reflectance for completely diffused illumination is obtained, however, by observing the sample at an angle of approximately 55 or $60^{\circ}$ from the normal. The degree of approximation obtained is shown in columns 9 and 10 of Table 2 . These data give the errors to be expected in the use of this type of reflectometer (for the measurement of $R_{\mathrm{D}}$ ), due entirely to the mixed specular and diffuse reflection characteristics of the samples.

The proper choice of the angle of observation is thus seen to be of prime importance; but, unfortunately, there is no one angle suitable for all samples and apparently no simple correction which may be readily applied to eliminate the effect.

\section{DESIGN OF SPHERE}

A properly designed sphere may, nevertheless, be of considerable use for many purposes. The data obtained suggest a design such as sketched in Figure 9. This sphere may be conveniently built up as shown in Figure 8, but first coated on the inner surface with a thick layer of white porcelain enamel (baked) and then smoked heavily with magnesium oxide. A surface of uniformly high reflectance would be obtained in this way.

The illuminated spot is located opposite the observed spot, and the beam of light is incident at approximately $50^{\circ}$. For this angle of 
incidence the first reflected light from the illuminated spot to the observed spot and thence to the photometer is approximately the same as it would be if the sphere wall were actually a perfect diffusor. The multiply reflected light, incident on both observed spot and sample, may be regarded as completely diffused.

The angle of observation may be varied over a range from 50 to $60^{\circ}$ (or more) by rotating the photometer (or the sphere) about an axis through the sample. The position of the observed spot and the angle at which it is viewed would vary somewhat, but the reflective properties of magnesium oxide are such that this variation would cause no appreciable change in the brightness of the observed spot.

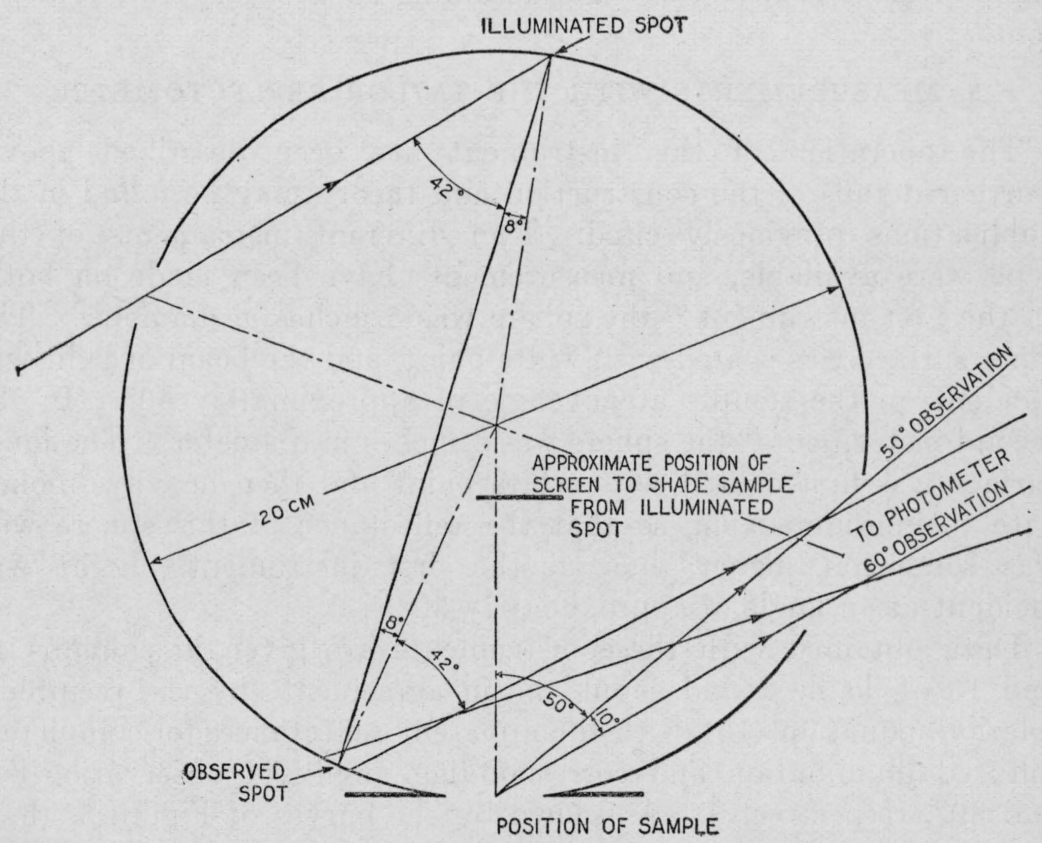

FIG. 9.-Suggested design of sphere

With this arrangement, the screen used to shade the sample from the illuminated spot will subtend a rather large angle at the sample. Care should be taken to make this screen of highly reflecting material, so that its brightness (as viewed from the sample) does not differ greatly from the brightness of the sphere wall. This is the essential condition to be fulfilled. The screen need not be opaque; a translucent screen, such as milk glass, of proper thickness, so that its brightness exactly matches the brightness of the sphere background, could be used. The openings into the sphere for the entrant and emergent beams of light would amount to about 1 per cent of the whole sphere surface. 
With this construction, it is believed that the error made in the evaluation of the total illumination on the sample would be very small if not negligible. Remaining imperfections, if they exist, could be fully compensated by correction factors, determined for the sphere as described above. Measurements on a sample over the range from 50 to $60^{\circ}$ would give some indication as to the uncertainty of the determination of the reflectance $R_{\mathrm{D}}$, and with some previous knowledge of the reflection characteristics of the sample this range of uncertainty could be narrowed by proper choice of the angle of observation. Thus, for matt samples of the kind herein studied (of not extremely low reflectance), one would be quite safe in choosing an angle of observation of $55^{\circ}$ and assuming an accuracy of 1 or 2 per cent.

\section{MEASUREMENTS WITH THE TAYLOR REFLECTOMETER}

The operation of this instrument has been described above. Further details of the construction and theory may be found in the publications previously cited. Two different instruments of this type were available, and measurements have been made on both. In the first instrument ${ }^{29}$ the sphere was 5 inches in diameter. The inner surface was coated with white paint, and the beam of light was incident on the sample at an angle of approximately $40^{\circ}$. In the second instrument ${ }^{30}$ the sphere was 4 inches in diameter. The inner surface was first coated with white paint and then heavily smoked with magnesium oxide, so that the reflectance of this sphere wall was somewhat higher than in the first instrument. Light was incident at an angle of approximately $30^{\circ}$.

Data obtained with these instruments are given in columns 14 and 15 of Table 2 and should be in agreement (by the reciprocal relation, equation (15), with the apparent reflectances for completely diffused illumination and corresponding angles of observation (40 and $30^{\circ}$, respectively). As shown by the curves of Figure 5, there is very little difference between the data for 40 and $30^{\circ}$. The values for $35^{\circ}$ are recorded in column 4 of Table 2 and are in good agreement with the sphere data - for samples of medium and high reflectance. Both instruments give definitely higher values, however, for samples of low reflectance.

An analysis of the performance of this type of reflectometer is not contemplated here. It may be noted, however, that in both instruments, when the entering beam is projected on the sphere wall,

20 This was the original instrument described by Taylor (loc. cit.) and located in the photometric laboratory of the Bureau of Standards. In the present work a Weber photometer was used for the brightness measurements instead of the Macbeth illuminometer, as described in the publications cited.

${ }^{30}$ This instrument was the property of the Research Laboratory of the Munsell Color Co., Baltimore, $\mathrm{Md}$., and kindly placed at the disposal of the writer for these measurements by the director of the laboratory. The photometric equipment consisted, essentially, of a flicker disk and milk glass diffusing screen, the brightness of which could be controlled by varying the distance of a lamp from the screen. 
the brightness of the observed spot varies appreciably with the position of the illuminated spot on the wall and with the reflectance of the sample itself, which occupies about 2.5 per cent of the sphere surface in the first instrument and 3.2 per cent in the second instrument.

A greater part of the apparatus used in this investigation was constructed in the physical laboratory shop of the Johns Hopkins University, the remainder in the shops of the Bureau of Standards. The writer wishes to express his appreciation of the help thereby extended by Professor Ames. The work was done at the Bureau of Standards as part of its research program in colorimetry.

Washington, October 29, 1927. 
